Pacific Journal of Mathematics

THE CAUCHY PROBLEM AND ASYMPTOTIC DECAY FOR
SOLUTIONS OF DIFFERENTIAL INEQUALITIES IN HILBERT
SPACE

Gerald Norman mile and MurRay Harold Potter 


\title{
THE CAUCHY PROBLEM AND ASYMPTOTIC DECAY FOR SOLUTIONS OF DIFFERENTIAL INEQUALITIES \\ IN HILBERT SPACE
}

\author{
G. N. Hile and M. H. Protter
}

1. Introduction. Let $H$ be a real or complex Hilbert space and $A$ an operator with domain $D$ in $H$. We consider the differential operators

$$
\begin{aligned}
& \frac{d u}{d t}-A u \\
& \frac{d^{2} u}{d t^{2}}-A u,
\end{aligned}
$$

and we investigate the Cauchy problem for differential equations and inequalities in which (1.1) and (1.2) are the principal parts. In general, we shall suppose that $A$ is a nonlinear, unbounded operator, neither symmetric nor antisymmetric, and dependent on $t$. In $\S \S 2$ and 3 we consider the case where $A$ is a linear operator.

Operators of the type in (1.1) were considered by Agmon and Nirenberg [1] who used a convexity argument to establish not only uniqueness theorems for the Cauchy problem but also maximal rates of decay as $t \rightarrow \infty$.

In $\S 2$ we treat linear operators $A$ which can be represented in the form $A=M+N$ where $M$ is symmetric and $N$ is antisymmetric. These hypotheses are used mainly for computational convenience. Instead of symmetry, the actual principal hypothesis on $M$. is the inequality

$$
\begin{gathered}
\frac{d}{d t} \operatorname{Re}(M(t) u(t), u(t))-2 \operatorname{Re}\left(M(t) u(t), u^{\prime}(t)\right) \\
\geqq-\gamma_{3}\|M(t) u(t)\|\|u(t)\|-\gamma_{4}\|u(t)\|^{2},
\end{gathered}
$$

where $\gamma_{3}, \gamma_{4}$ are positive constants. Thus the results of Section 2, when applied to differential operators $A$, are not restricted to those operators for which the principal part is self-adjoint. Furthermore, the condition of antisymmetry on $N$ is easily relaxed. The arguments in $\S 2$ are applicable almost without change if $N$ satisfies either the inequality

$$
\operatorname{Re}(N(t) u(t), u(t)) \leqq \gamma(t)\|u(t)\|^{2}
$$

or

$$
\operatorname{Re}(N(t) u(t), u(t)) \geqq-\gamma(t)\|u(t)\|^{2}
$$


for some positive function $\gamma=\gamma(t)$. For computational convenience the theorems in Section 2 will be established for the case $M$ symmetric and $N$ antisymmetric. The differential inequality we investigate is of the type

$$
\left\|\frac{d u}{d t}(t)-A(t) u(t)\right\|^{2} \leqq \gamma(t)\left[\omega(t)+\int_{0}^{t} \omega(s) d s\right]
$$

where

$$
\omega(t)=\|u(t)\|^{2}+\|M(t) u(t)\|\|u(t)\|,
$$

and $\gamma$ is a prescribed function of $t$. We note that (1.4) is more than a customary semi-linear inequality since $M$, the symmetric part of $A$, occurs on the right side. In the cases which occur most frequently, that is when $A$ is a partial differential operator, the part $M$ corresponds to the principal part of the differential operator while $N$ frequently corresponds to the terms of lower order. Thus the inequality (1.4) allows the differential operator (1.1) to be estimated in terms of the norm of its principal part. In the paper [1], Agmon and Nirenberg decompose $A$ into the sum of three operators, of which one is symmetric and the other two are antisymmetric. While this decomposition is more general than the one we employ of a decomposition into one symmetric and one antisymmetric operator, the broader class of inequalities (1.4) which we treat, together with the conditions we impose on $M$ and $N$, enables us to include the uniqueness results in [1] as a special case. The method we employ is one of weighted $L_{2}$-estimates, a technique that was developed for differential operators in [15] and extended by Murray [10], Murray and Protter [12], and Ogawa [13, 14].

In a series of papers Levine [5-9] employed the convexity method to study the Cauchy problem for broad classes of first order and second order differential inequalities. He considered principal parts of the form $P(d u / d t)-A u$ and $P\left(d^{2} u / d t^{2}\right)+Q(d u / d t)-A u$ where $P$ and $Q$ are also linear operators which depend on $t$. The results he obtains overlap with ours, and it is not likely that the weighted $L_{2}$-method which we employ can be applied to these more general operators without making some unusual assumptions about the interaction of the operators $P$ and $Q$ with $A$ - assumptions not required in the convexity method.

A detailed analysis of the asymptotic behavior of solutions of equations and inequalities has been made by similar methods for partial differential operators. Murray [11] considered ultrahyperbolic operators, Ogawa [14] discussed hyperbolic operators, Knops and Payne [3,4], and Knops, Levine and Payne [2] investigated 
both the abstract problem and its applications to elasticity.

In $\S 3$ we consider second order inequalities of the type

$$
\left\|\frac{d^{2} u}{d t^{2}}-A u\right\|^{2} \leqq \gamma\left[\mu(t)+\int_{0}^{t} \mu(s) d s\right]
$$

where

$$
\mu(t)=\|u(t)\|^{2}+\left\|u^{\prime}(t)\right\|^{2}+|(M(t) u(t), u(t))|
$$

and $A=M+N$. We establish the uniqueness of solutions of the Cauchy problem under appropriate conditions on $M$ and $N$.

For both first and second order operators we show that solutions which exist for all $t>0$ cannot decay too rapidly as $t \rightarrow \infty$ without being identically zero.

In $\S \S 4$ and 5 first and second order inequalities with nonlinear operators are considered. In the first order case, we show that the usual convexity argument can be modified to treat inequalities of the form

$$
\left\|\frac{d u}{d t}-A u\right\|^{2} \leqq \gamma_{1}\|u\|^{2}+\gamma_{2}|\operatorname{Re}(A u, u)|,
$$

where an appropriate one-sided bound is placed on $\operatorname{Re}(A u, u)$. The persistence of the zero solution of the Cauchy problem is shown and maximal rates of decay are obtained. If $A$ satisfies a differentiability condition of the form (1.3) (with $M$ replaced by $A$ ), then we may perturb $A$ by another nonlinear operator $B$, with appropriate one-sided bounds on $\operatorname{Re}(B u, u)$ and $\operatorname{Re}(A u, B u)$, and obtain analogous results for the inequality

$$
\frac{d u}{d t}-A u-B u\left\|^{2} \leqq \gamma_{1}\right\| u\left\|^{2}+\gamma_{2}\right\| A u\|\| u \| .
$$

In the second order case we obtain similar results for inequalities of the form

$$
\left\|\frac{d^{2} u}{d t^{2}}-A u-B u\right\|^{2} \leqq \gamma_{1}\|u\|^{2}+\gamma_{2}\left\|u^{\prime}\right\|^{2}+\gamma_{3}|\operatorname{Re}(A u, u)| .
$$

2. First order linear operators. Let $H$ be a complex Hilbert space with the usual inner product and norm. We shall consider functions

$$
u:[0, T) \longrightarrow H
$$

with $T=+\infty$ allowed. The strong derivative of $u$,

$$
u^{\prime}(t)=\lim _{h \rightarrow 0} \frac{1}{h}[u(t+h)-u(t)],
$$


where the limit is taken in the Hilbert space norm, is assumed to exist for all $t$ in $(0, T)$.

We shall suppose throughout this section that $u$ satisfies the following two conditions:

(i ) $u \in C([0, T) ; H) \cap C^{1}((0, T) ; H)$

(ii) $\left\|u^{\prime}(t)\right\| \leqq K$ on $(0, T), K$ a constant.

Let $A=A(t)$ be a linear operator on $H$ for each $t \in(0, T)$ which, in general, will be unbounded. We shall suppose that $A$ can be decomposed

$$
A(t)=M(t)+N(t)
$$

where $M$ is symmetric and $N$ anti-symmetric with respect to the inner product of $H$. We assume that $D_{t}$, the domain of $M(t)$ and $N(t)$, is a linear manifold in $H$.

We shall be concerned with the Cauchy problem for the differential operator

$$
L u \equiv u^{\prime}-A u \equiv u^{\prime}-M u-N u
$$

for $u(t) \in D_{t}$ with mild restrictions on the operator $A$. The first hypothesis concerns the relationship between $M$ and $N$. We assume

$$
\operatorname{Re}(M(t) w, N(t) w) \geqq-\gamma_{1}\|M(t) w\| \cdot\|w\|-\gamma_{2}\|w\|^{2}
$$

for all $t \in(0, T)$ and $w \in D_{t} ; \gamma_{1}$ and $\gamma_{2}$ are positive constants. We note that if $A$ is either symmetric or antisymmetric, then (I) is automatically satisfied. Furthermore we assume that $u(t) \in D_{t}, t \in$ $(0, T)$, that $M u, N u \in C((0, T) ; H)$, and that $\|M(t) u(t)\|,\|N(t) u(t)\| \leqq$ $K$ on $(0, T)$.

The second hypothesis concerns only the action of the symmetric part of $A$ on functions $u$ which satisfy the differential inequality to be treated. We suppose

$$
\begin{aligned}
& \frac{d}{d t}(M(t) u(t), u(t))-2 \operatorname{Re}\left(M(t) u(t), u^{\prime}(t)\right) \\
& \geqq-\gamma_{3}\|M(t) u(t)\|\|u(t)\|-\gamma_{4}\|u(t)\|^{2}
\end{aligned}
$$

where $\gamma_{3}$ and $\gamma_{4}$ are positive constants. Of course, (II) implies that $(M u, u)$ is differentiable, and we shall suppose for convenience that $(d / d t)(M u, u)$ is continuous on $(0, T)$.

The following weighted $L_{2}$ inequality for the operator $L$ acting on functions with compact support is basic.

Lemma 1. Suppose $0<T_{0}<1$ and $t_{0}>0$ is such that $t_{0}+T_{0}<$ 1. Assume that $u(t)$ has compact support on $[0, T]$. Then for all sufficiently large $\beta>0$, the size depending only on $\gamma_{i}, i=1, \cdots, 4$, 
the following inequality holds:

$$
\begin{gathered}
\beta^{2} \int_{0}^{T_{0}} \tau^{-\beta-2} e^{2 \tau^{-\beta}}\|u\|^{2} d t+C_{0} \int_{0}^{T_{0}} \tau^{\beta} e^{2 \tau^{-\beta}}\|M u\|^{2} d t \\
\quad \leqq C_{1} \int_{0}^{T_{0}} e^{2 \tau-\beta}\|L u\|^{2} d t
\end{gathered}
$$

where $\tau=t+t_{0}$ and $C_{0}, C_{1}$ are absolute constants.

Proof. For convenience we set $\phi(t)=-\left(t+t_{0}\right)^{-\beta}$ and define $v=e^{-\phi} u$. Then

$$
L u=e^{\phi}\left[v^{\prime}+\phi^{\prime} v-M v-N v\right],
$$

and defining $\alpha=\alpha(t)$ a continuous function from $[0, T)$ to $R^{1}$ to be chosen later, we have

$$
e^{-2 \phi}\|L u\|^{2}=\left\|v^{\prime}+\phi^{\prime} v-\alpha M v-(1-\alpha) M v-N v\right\|^{2} .
$$

Therefore, integrating with respect to $t$ from 0 to $T_{0}$, we find

$$
\begin{aligned}
\int e^{-2 \phi}\|L u\|^{2} \geqq 2 \operatorname{Re} \int\left(v^{\prime}-\alpha M v-N v, \phi^{\prime} v-(1-\alpha) M v\right) \\
=2 \operatorname{Re} \int \phi^{\prime}\left(v^{\prime}, v\right)+2 \int \alpha(1-\alpha)\|M v\|^{2}-2 \int \alpha \phi^{\prime}(M v, v) \\
-2 \operatorname{Re} \int(1-\alpha)\left(v^{\prime}, M v\right)+2 \operatorname{Re} \int(1-\alpha)(N v, M v) \\
\equiv I_{1}+\cdots+I_{5} .
\end{aligned}
$$

We estimate each of the integrals $I_{i}$ :

$$
I_{1}=\int\left(\phi^{\prime}\|v\|^{2}\right)^{\prime}-\int \phi^{\prime \prime}\|v\|^{2}=-\int \phi^{\prime \prime}\|v\|^{2} .
$$

We now restrict the choice of $\alpha(t)$ so that $0<\alpha<1 / 2$. Hence

$$
I_{2}=2 \int \alpha(1-\alpha)\|M v\|^{2} \geqq \int \alpha\|M v\|^{2} .
$$

We use the Cauchy inequality in $I_{3}$ to obtain

$$
I_{3}=-2 \int\left(M v, \alpha \phi^{\prime} v\right) \geqq-\int \delta\|M v\|^{2}-\int \frac{1}{\delta} \alpha^{2} \phi^{\prime 2}\|v\|^{2}
$$

for any $\delta>0$. For $I_{4}$ we employ hypothesis (II) to get

$$
\begin{aligned}
I_{4} & =-2 \operatorname{Re} \int(1-\alpha)\left(v^{\prime}, M v\right)=-2 \operatorname{Re} \int(1-\alpha) e^{-2 \phi}\left(u^{\prime}-\phi^{\prime} u, M u\right) \\
& =-2 \operatorname{Re} \int(1-\alpha) e^{-2 \phi}\left(u^{\prime}, M u\right)+2 \int(1-\alpha) e^{-2 \phi} \phi^{\prime}(u, M u)
\end{aligned}
$$




$$
\begin{aligned}
\geqq & \int(1-\alpha) e^{-2 \phi}\left[-\frac{d}{d t}(M u, u)-\gamma_{3}\|M u\| \cdot\|u\|-\gamma_{4}\|u\|^{2}\right] \\
& +2 \int(1-\alpha) e^{-2 \phi} \phi^{\prime}(u, M u) \\
= & \int\left\{-\frac{d}{d t}\left[(1-\alpha) e^{-2 \phi}(M u, u)\right]-\alpha^{\prime} e^{-2 \phi}(M u, u)\right. \\
& -2(1-\alpha) \phi^{\prime} e^{-2 \phi}(M u, u)-\gamma_{3}(1-\alpha) e^{-2 \phi}\|\| M u\|\cdot\| u \| \\
& \left.-\gamma_{4}(1-\alpha) e^{-2 \phi}\|u\|^{2}\right\}+2 \int(1-\alpha) e^{-2 \phi} \phi^{\prime}(u, M u) \\
= & \int\left[-\alpha^{\prime}(M v, v)-\gamma_{3}(1-\alpha)\|M v\| \cdot\|v\|-\gamma_{4}(1-\alpha)\|v\|^{2}\right] .
\end{aligned}
$$

Using the Cauchy inequality again, we find

$$
I_{4} \geqq \int\left[-d_{1}\|M v\|^{2}-d_{2}\|v\|^{2}\right]
$$

where

$$
\begin{aligned}
& d_{1}=\frac{1}{2}\left(\left|\alpha^{\prime}\right| \varepsilon_{1}+(1-\alpha) \gamma_{3} \varepsilon_{2}\right) \\
& d_{2}=\frac{1}{2}\left(\frac{1}{\varepsilon_{1}}\left|\alpha^{\prime}\right|+\frac{1}{\varepsilon_{2}}(1-\alpha) \gamma_{3}+2 \gamma_{4}(1-\alpha)\right)
\end{aligned}
$$

and $\varepsilon_{1}, \varepsilon_{2}$ are arbitrary positive quantities. We employ (I) to estimate $I_{5}$ :

$$
I_{5} \geqq \int-d_{3}\|M v\|^{2}-d_{4}\|v\|^{2}
$$

where

$$
d_{3}=\gamma_{1}(1-\alpha) \varepsilon_{3}, d_{4}=\gamma_{1}(1-\alpha) \frac{1}{\varepsilon_{3}}+2 \gamma_{2}(1-\alpha)
$$

and $\varepsilon_{3}$ is an arbitrary positive quantity. Combining the estimates for $I_{1}, \cdots, I_{5}$, we obtain

$$
\begin{aligned}
\int e^{-2 \phi}\|L u\|^{2} \geqq & \int\|v\|^{2}\left[-\phi^{\prime \prime}-\frac{1}{\delta} \alpha^{2} \phi^{\prime 2}-d_{2}-d_{4}\right] \\
& +\|M v\|^{2}\left[\alpha-\delta-d_{1}-d_{3}\right] .
\end{aligned}
$$

We choose $\delta=d_{1}=d_{3}=\alpha / 4$. Then the coefficient of $\|M v\|^{2}$ is $(1 / 4)(\alpha)$. Also, we select $\varepsilon_{1}=(1 / 4) \alpha /\left|\alpha^{\prime}\right|, \varepsilon_{2}=(1 / 4) \alpha / \gamma_{3}(1-\alpha), \varepsilon_{3}=$ $(1 / 4) \alpha / \gamma_{1}(1-\alpha)$. Finally, we set

$$
\alpha=k_{0} \tau^{\beta},
$$

and taking into account the values $\phi^{\prime \prime}=-\beta(\beta+1) \tau^{-\beta-2},{\phi^{\prime 2}}^{2}=\beta^{2} \tau^{-2 \beta-2}$ 
we get, for sufficiently small $k_{0}$ and sufficiently large $\beta$, the coefficient of $\|v\|^{2}$ in the form

$$
k_{1} \beta^{2} \tau^{-\beta-2}
$$

where $k_{1}$ is a numerical constant, and clearly $\beta$ depends only on the $\gamma_{i}$.

We now consider functions $u$ which satisfy a differential inequality of the form

$$
\|(L u)(t)\|^{2} \leqq \gamma\left[\omega(t)+\int_{0}^{t} \omega(s) d s\right], \quad t \in(0, T)
$$

where $L$ is given by (2.1) and

$$
\omega(t)=\|u(t)\|^{2}+\|M(t) u(t)\| \cdot\|u(t)\| .
$$

THEOREM 1. Suppose condition (I) holds, and $u$ satisfies (II) and is a solution of (2.4). If $u(0)=0$, then $u \equiv 0$ on $[0, T)$.

Proof. We shall show that $u \equiv 0$ on $\left[0, T_{0}\right)$ for sufficiently small $T_{0}$. Then a step-by-step procedure establishes the result on $[0, T)$. Let $\varepsilon>0$ be given and define the $C^{\infty}$ function $\zeta(t)=1$ for $0 \leqq t \leqq T_{0}-\varepsilon$, =0 for $t \geqq T_{0}$, and such that $0<\zeta<1$ for $T_{0}-\varepsilon<$ $t<T_{0}$.

The function $\zeta u$ satisfies property (II), since

$$
\begin{aligned}
\frac{d}{d t}(M & (\zeta u), \zeta u)-2 \operatorname{Re}\left(M(\zeta u),(\zeta u)^{\prime}\right) \\
& =\frac{d}{d t} \zeta^{2}(M u, u)-2 \zeta^{2} \operatorname{Re}\left(M u, u^{\prime}\right)-2 \zeta \zeta^{\prime}(M u, u) \\
& =\zeta^{2}\left[\frac{d}{d t}(M u, u)-2 \operatorname{Re}\left(M u, u^{\prime}\right)\right] \\
& \geqq-\zeta^{2}\left[\gamma_{3}\|M u\|\|u\|+\gamma_{4}\|u\|^{2}\right] \\
& =-\gamma_{3}\|M(\zeta u)\|\|\zeta u\|-\gamma_{4}\|\zeta u\|^{2} .
\end{aligned}
$$

Properties (i), (ii), and the other hypotheses of Lemma 1 are easily verified for $\zeta u$. Hence we may apply Lemma 1 to the function $\zeta u$ to obtain

$$
\begin{aligned}
\beta^{2} \int_{0}^{T_{0}-\varepsilon} \tau^{-\beta-2} e^{2 \tau-\beta}\|u\|^{2} d t+C_{0} \int_{0}^{T_{0}-\varepsilon} \tau^{\beta} e^{2 \tau-\beta}\|M u\|^{2} d t \\
\quad \leqq C_{1} \int_{0}^{T_{0}-\varepsilon} e^{2 \tau^{-\beta}}\|L u\|^{2} d t+C_{1} \int_{T_{0}-\varepsilon}^{T_{0}} e^{2 \tau-\beta}\|L(\zeta u)\|^{2} d t .
\end{aligned}
$$

From (2.4) we find 


$$
\begin{aligned}
& \int_{0}^{T_{0}-\varepsilon} e^{2 \tau^{-\beta}}\|L u\|^{2} d t \leqq \gamma \int_{0}^{T_{0}-\varepsilon} e^{2 \tau-\beta}\left(\omega(t)+\int_{0}^{t} \omega(s) d s\right) d t \\
& \leqq \gamma \int_{0}^{T_{0}-\varepsilon} e^{2 \tau^{-\beta}} \omega(t) d t+\gamma \int_{0}^{T_{0}-\varepsilon} e^{2 \tau-\beta} \int_{0}^{t} \omega(s) d s d t \\
& =\gamma \int_{0}^{T_{0}-\varepsilon} e^{2 \tau-\beta} \omega(t) d t+\gamma \int_{0}^{T_{0}-\varepsilon} \int_{s}^{T_{0}-\varepsilon} \omega(s) e^{2 \tau-\beta} d t d s .
\end{aligned}
$$

Since $\tau<1$, we have $2 \beta \tau^{-\beta-1}>1$ and so

$$
\begin{aligned}
\gamma \int_{0}^{T_{0}-\varepsilon} \int_{s}^{T_{0}-\varepsilon} \omega(s) e^{2 \tau-\beta} d t d s & \leqq \gamma \int_{0}^{T_{0}-\varepsilon} \int_{s}^{T_{0}-\varepsilon} 2 \beta \tau^{-\beta-1} \omega(s) e^{2 \tau-\beta} d t d s \\
& \leqq \gamma \int_{0}^{T_{0}-\varepsilon} \omega(s) e^{2\left(s+t_{0}\right)-\beta} d s
\end{aligned}
$$

Hence

$$
\begin{gathered}
\int_{0}^{T_{0}-\varepsilon} e^{2 \tau-\beta}\|L u\|^{2} d t \leqq 2 \gamma \int_{0}^{T_{0}-\varepsilon} e^{2 \tau^{-\beta}} \omega(t) d t \\
\leqq 2 \gamma \int_{0}^{T_{0}-\varepsilon} e^{2 \tau-\beta}\left[\|u(t)\|^{2}+\|M(t) u(t)\| \cdot\|u(t)\|\right] d t \\
\leqq \int_{0}^{T_{0}-\varepsilon} e^{2 \tau-\beta}\left[2 \gamma\|u(t)\|^{2}+k_{2} \tau^{\beta+1}\|M(t) u(t)\|^{2}\right. \\
\left.\quad+\frac{1}{k_{2}} \tau^{-\beta-1} \gamma^{2}\|u(t)\|^{2}\right] d t \\
\leqq k_{2} \int_{0}^{T_{0}-\varepsilon} e^{2 \tau^{-\beta}} \tau^{\beta+1}\|M(t) u(t)\|^{2} d t \\
\quad+\int_{0}^{T_{0}-\varepsilon} e^{2 \tau-\beta}\left[2 \gamma+\frac{1}{k_{2}} \tau^{-\beta-1} \gamma^{2}\right]\|u(t)\|^{2} d t
\end{gathered}
$$

Thus for $\beta$ sufficiently large, $k_{2}$ a sufficiently small constant, and $\bar{C}_{1}=2 C_{1}$ we find

$$
\beta^{2} \int_{0}^{T_{0}-\varepsilon} e^{2 \tau-\beta} \tau^{-\beta-2}\|u(t)\|^{2} d t \leqq \bar{C}_{1} \int_{T_{0}-\varepsilon}^{T_{0}} e^{2 \tau-\beta}\|L(\zeta u)\|^{2} d t
$$

Since $e^{2 \tau^{-\beta}} \tau^{-\beta-2} \geqq e^{2\left(T_{0}-\varepsilon\right)-\beta}$ on $\left[0, T_{0}-\varepsilon\right]$ and

$$
e^{2 \tau^{-\beta}} \leqq e^{2\left(T_{0}-\varepsilon\right)-\beta} \text { on }\left[T_{0}-\varepsilon, T_{0}\right] \text {, }
$$

we have

$$
\beta^{2} \int_{0}^{T_{0}-\varepsilon}\|u(t)\|^{2} d t \leqq \bar{C}_{1} \int_{T_{0}-\varepsilon}^{T_{0}}\left\|L(\zeta u)^{2}\right\| d t
$$

Letting $\beta \rightarrow+\infty$ we find $u \equiv 0$ on $\left[0, T_{0}-\varepsilon\right)$. Since $\varepsilon$ is arbitrary we have $u \equiv 0$ on $\left[0, T_{0}\right)$.

We may repeat the process on an interval of length less than 
1 beginning at $T_{0}$ and thus proceed in a step-by-step manner to conclude that $u \equiv 0$ on $[0, T)$.

The method used to obtain Lemma 1 may be adapted to yield results on the asymptotic behavior of solutions of (2.1) as $t \rightarrow+\infty$. For this purpose we assume that $\gamma_{i}, i=1,2,3,4$ in (I) and (II) now depend on $t$.

The following inequality is a modification of Lemma 1.

Lemma 2. Suppose that $u(1)=0$. Then there are positive constants $k_{2}, k_{3}, k_{4}$ such that for sufficiently large $\beta>0$ and all $T>1$, the following inequality holds:

$$
\begin{aligned}
& \int_{1}^{T} k_{2} t^{-\beta} e^{2 t^{\beta}}\|M(t) u(t)\|^{2} d t+\int_{1}^{T}\left[k_{3} \beta^{2} t^{\beta-2}-k_{4} t^{\beta}\left(\gamma_{1}(t)^{2}+\gamma_{3}(t)^{2}\right)\right. \\
& \left.-2 \gamma_{2}(t)-\gamma_{4}(t)\right] e^{2 t^{\beta}}\|u(t)\|^{2} d t-\left(1-4 k_{2} T^{-\beta}\right) e^{2 T^{\beta}}(M(T) u(T), u(T)) \\
& -\beta T^{\beta-1} e^{2 T^{\beta}}\|u(T)\|^{2} \leqq \int_{1}^{T} e^{2 t^{\beta}}\|L u(t)\|^{2} d t
\end{aligned}
$$

Proof. We proceed as in the proof of Lemma 1, but choose $\phi$ and $\alpha$ differently. The integrals $I_{1}, \cdots, I_{5}$ are defined as before, and we suppose that $\alpha=\alpha(t)$ is such that $0<\alpha<1 / 2$. Then if (2.3) is integrated between 1 and $T$, we have

$$
\begin{aligned}
& I_{1}=\phi^{\prime}(T)\|v(T)\|^{2}-\int_{1}^{T} \phi^{\prime \prime}\|v(t)\|^{2} d t \\
& I_{2}=2 \int_{1}^{T} \alpha(1-\alpha)\|M v(t)\|^{2} d t \geqq \int_{1}^{T} \alpha\|M v\|^{2} d t \\
& I_{3} \geqq-\int_{1}^{T} \delta\|M v\|^{2} d t-\int_{1}^{T} \delta^{-1} \alpha^{2} \phi^{\prime 2}\|v\|^{2} d t \\
& I_{4} \geqq-[1-\alpha(T)](M(T) v(T), v(T))-\int_{1}^{T}\left[d_{1}\|M v\|^{2}+d_{2}\|v\|^{2}\right] d t \\
& I_{5} \geqq \int_{1}^{T}\left[-d_{3}\|M v\|^{2}-d_{4}\|v\|^{2}\right] d t
\end{aligned}
$$

where

$$
\begin{aligned}
& d_{1}=\frac{1}{2}\left(\left|\alpha^{\prime}\right| \varepsilon_{1}+(1-\alpha) \gamma_{3} \varepsilon_{2}\right) \\
& d_{2}=\frac{1}{2}\left(\frac{1}{\varepsilon_{1}}\left|\alpha^{\prime}\right|+\frac{1}{\varepsilon_{2}} \gamma_{3}(1-\alpha)+2 \gamma_{4}(1-\alpha)\right) \\
& d_{3}=\gamma_{1}(1-\alpha) \varepsilon_{3}, d_{4}=\gamma_{1}(1-\alpha) \frac{1}{\varepsilon_{3}}+2 \gamma_{2}(1-\alpha)
\end{aligned}
$$

and $\varepsilon_{1}, \varepsilon_{2}, \varepsilon_{3}$ are arbitrary positive numbers. Thus we find 


$$
\begin{aligned}
\int_{1}^{T} e^{-2 \phi} & \|L u\|^{2} d t \geqq \int_{1}^{T}\|v\|^{2}\left[-\phi^{\prime \prime}-\delta^{-1} \alpha^{2} \phi^{\prime 2}-d_{2}-d_{4}\right] d t \\
& +\int_{1}^{T}\|M v\|^{2}\left[\alpha-\delta-d_{1}-d_{3}\right] d t+\phi^{\prime}(T)\|v(T)\|^{2} \\
& -[1-\alpha(T)](M(T) v(T), v(T)) .
\end{aligned}
$$

We make the selections

$$
\dot{\phi}=-t^{\beta}, \alpha=4 k_{2} t^{-\beta}
$$

with $k_{2}$ a constant, $0<k_{2} \leqq 1 / 8$, so that $0<\alpha \leqq 1 / 2$ for $t \geqq 1$. We choose $\delta=d_{1}=d_{3}=\alpha / 4, \varepsilon_{1}=(1 / 4)\left(\alpha /\left|\alpha^{\prime}\right|\right), \varepsilon_{2}=(1 / 4)\left(\alpha / \gamma_{3}(1-\alpha)\right), \varepsilon_{3}=$ $(1 / 4)\left(\alpha / \gamma_{1}(1-\alpha)\right)$. Then $(2.8)$ becomes

$$
\begin{aligned}
\int_{1}^{T} e^{2 t^{\beta}} & \|L u\|^{2} d t \geqq k_{2} \int_{1}^{T} t^{-\beta} e^{2 t^{\beta}}\|M u\|^{2} d t \\
& +\int_{1}^{T} e^{2 t^{\beta}}\|u\|^{2}\left[\left(\beta^{2}-16 k_{2} \beta^{2}-\beta\right) t^{\beta-2}-\frac{1}{2 k_{2}}(1-\alpha)^{2}\left(\gamma_{3}^{2}+2 \gamma_{1}^{2}\right) t^{\beta}\right. \\
& \left.-(1-\alpha)\left(2 \gamma_{2}+\gamma_{4}\right)-8 k_{2} \beta^{2} t^{-\beta-2}\right] d t-\beta T^{\beta-1} e^{2 T^{\beta}}\|u(T)\|^{2} \\
& -[1-\alpha(T)] e^{2 T^{\beta}}(M(T) u(T), u(T)) .
\end{aligned}
$$

We now select $k_{2}$ so small and $\beta$ so large that

$$
\beta^{2}-\beta-16 k_{2} \beta^{2}-8 k_{2} \beta^{2} t^{-\beta-2}>k_{3} \beta^{2} \text { for all } t \geqq 1
$$

and some positive constant $k_{3}$; then inequality (2.7) is immediate.

We shall show that solutions of differential inequalities similar to (2.4) cannot decay too rapidly as $t \rightarrow+\infty$ without being identically zero. We consider the inequality

$$
\|(L u)(t)\|^{2} \leqq \gamma_{0}(t)\|u(t)\|^{2}+\gamma_{5}(t)\|M(t) u(t)\|\|u(t)\| .
$$

We assume that $u$ is a solution of (2.9) for all $t>0$ which for all $\beta>0$ satisfies

$$
\begin{aligned}
& \lim _{t \rightarrow \infty} e^{2 t^{\beta}}\|u(t)\|^{2}=0 . \\
& \varlimsup_{t \rightarrow \infty} e^{2 t^{\beta}}(M(t) u(t), u(t)) \leqq 0 .
\end{aligned}
$$

Furthermore, we shall suppose that

$$
\begin{aligned}
& \gamma_{1}(t), \gamma_{3}(t), \gamma_{5}(t) \text { are } O\left(t^{-1}\right) \text { as } t \rightarrow \infty \\
& \gamma_{0}(t), \gamma_{2}(t), \gamma_{4}(t) \text { are } O\left(t^{n}\right) \text { as } t \rightarrow \infty \text { for some } n \geqq 0 \\
& \gamma_{i}, i=0,1, \cdots, 5, \text { are bounded on compact subsets of }(0, \infty) .
\end{aligned}
$$

The asymptotic behavior of $u$ is described in the following result. Since no conditions on $u\left(0^{+}\right)$are required, we relax conditions (i) 
and (ii) by requiring only that $u \in C^{1}((0, \infty) ; H)$.

THEOREM 2. Suppose that $u$ is a solution of (2.9) for all $t>0$, that $u \in C^{1}((0, \infty) ; H)$, and that (I) and (II) hold. Let $\gamma_{i}, i=0,1, \cdots, 5$, satisfy (2.11). If, in addition, $u$ satisfies (2.10), then $u \equiv 0$.

Proof. We will show that $u \equiv 0$ on $\left[t_{0}, \infty\right)$ for each $t_{0}>0$. It suffices to establish this fact for $t_{0}=2$, since the general case involves only a change of scale which leaves the form of our hypotheses unchanged.

Let $\zeta(t)$ be a $C^{\infty}$ function such that $\zeta=0$ for $0 \leqq t \leqq 1, \zeta=1$ for $t \geqq 2$, and $0 \leqq \zeta \leqq 1$ for $1 \leqq t \leqq 2$. Define $v(t)=\zeta(t) u(t)$. Then from Lemma 2 we have for any $T>2$,

$$
\begin{aligned}
& k_{2} \int_{1}^{2} t^{-\beta} e^{2 t^{\beta}}\|M v\|^{2}+k_{2} \int_{2}^{T} t^{-\beta} e^{2 t^{\beta}}\|M u\|^{2} \\
& \quad+\int_{1}^{T}\left[k_{3} \beta^{2} t^{\beta-2}-k_{4} t^{\beta}\left(\gamma_{1}^{2}+\gamma_{3}^{2}\right)-2 \gamma_{2}-\gamma_{4}\right] e^{2 t \beta}\|v(t)\|^{2} d t \\
& \quad-\left(1-4 k_{2} T^{-\beta}\right) e^{2 T^{\beta}}(M(T) u(T), u(T))-\beta T^{\beta-1} e^{2 T^{\beta}} \| u\left(T \|^{2}\right. \\
& \leqq \int_{1}^{2} e^{2 t^{\beta}}\|L v\|^{2}+\int_{2}^{T} e^{2 t^{\beta}}\|L u\|^{2} .
\end{aligned}
$$

Since $\gamma_{i}, i=1, \cdots, 4$, are locally bounded, the integrals on the left from 1 to 2 may be dropped if $\beta$ is sufficiently large, thus strengthening the inequality. Now using (2.9) we obtain

$$
\begin{aligned}
k_{2} \int_{2}^{T} t^{-\beta} e^{2 t \beta}\|M u\|^{2} & \\
& +\int_{2}^{T}\left[k_{3} \beta^{2} t^{\beta-2}-k_{4} t^{\beta}\left(\gamma_{1}^{2}+\gamma_{3}^{2}\right)-2 \gamma_{2}-\gamma_{4}\right] e^{2 t^{\beta}}\|u\|^{2} \\
& -\left(1-4 k_{2} T^{-\beta}\right) e^{2 T^{\beta}}(M(T) u(T), u(T))-\beta T^{\beta-1} e^{2 T^{\beta}}\|u(T)\|^{2} \\
\leqq & \int_{1}^{2} e^{2 t^{\beta}}\|L v\|^{2}+\int_{2}^{T} e^{2 t \beta}\left[\gamma_{0}\|u\|^{2}+\gamma_{5}\|M u\|\|u\|\right] .
\end{aligned}
$$

With $\theta$ any positive constant we substitute the inequality

$$
\|M u\|\|u\| \leqq \frac{1}{2} \theta t^{-\beta+1}\|M u\|^{2}+\frac{1}{2 \theta} t^{\beta-1}\|u\|^{2}
$$

into (2.12) getting

$$
\begin{aligned}
\int_{2}^{T} e^{2 t^{\beta}} \| & M u \|^{2}\left[k_{2} t^{-\beta}-\frac{1}{2} \theta \gamma_{5} t^{-\beta+1}\right] \\
& +\int_{2}^{T} e^{2 t^{\beta}}\|u\|^{2}\left[k_{3} \beta^{2} t^{\beta-2}-k_{4} t^{\beta}\left(\gamma_{1}^{2}+\gamma_{3}^{2}\right)-2 \gamma_{2}-\gamma_{4}-\frac{1}{2 \theta} \gamma_{5} t^{\beta-1}-\gamma_{0}\right] \\
\leqq & \int_{1}^{2} e^{2 t^{\beta}}\|L v\|^{2}+\left(1-4 k_{2} T^{-\beta}\right) e^{2 T^{\beta}}(M(T) u(T), u(T))+\beta T^{\beta-1} e^{2 T^{\beta}}\|u(T)\|^{2}
\end{aligned}
$$


The hypotheses (2.11) imply that there is a constant $c>0$ such that

$$
\begin{aligned}
& \left|\gamma_{i}(t)\right| \leqq c t^{-1} \text { for } t \geqq 2, i=1,3,5 \\
& \left|\gamma_{i}(t)\right| \leqq c t^{n} \text { for } t \geqq 2, i=0,2,4 .
\end{aligned}
$$

Hence first choosing $\theta$ sufficiently small and then choosing $\beta$ sufficiently large, we find

$$
\begin{aligned}
e^{2^{\beta+1}} \int_{2}^{T} & \|M u\|^{2} t^{-\beta}\left[k_{2}-\frac{1}{2} \theta c\right] \\
& +e^{2 \beta+1} \int_{2}^{T}\|u\|^{2}\left[t^{\beta-2}\left(k_{3} \beta^{2}-2 k_{4} c^{2}-\frac{c}{2 \theta}\right)-4 c t^{n}\right] \\
\leqq & e^{2 \beta+1} \int_{1}^{2}\|L v\|^{2}+\left(1-4 k_{2} T^{-\beta}\right) e^{2 T^{\beta}}(M(T) u(T), u(T)) \\
& +\beta T^{\beta-1} e^{2 T^{\beta}}\|u(T)\|^{2} .
\end{aligned}
$$

Discarding the first integral on the left, we find that for $\beta$ sufficiently large,

$$
\begin{aligned}
& 2^{\beta-3} k_{3} \beta^{2} \int_{2}^{T}\|u\|^{2} \leqq \int_{1}^{2}\|L v\|^{2} \\
& \quad+e^{-2^{\beta+1}}\left[\left(1-4 k_{2} T^{-\beta}\right) e^{2 T^{\beta}}(M(T) u(T), u(T))+\beta T^{\beta-1} e^{2 T^{\beta}}\|u(T)\|^{2}\right] .
\end{aligned}
$$

Because of (2.10), we have $\beta T^{\beta-1} e^{2 T^{\beta}}\|u(T)\|^{2} \rightarrow 0$ as $T \rightarrow \infty$ since $\beta T^{\beta-1} e^{2 T^{\beta}} \leqq e^{2 T^{\beta+1}}$ for all sufficiently large $T$. Also, since $0<4 k_{2} T^{-\beta}<$ 1 , we find from (2.10) that

$$
\varlimsup_{T \rightarrow \infty}\left(1-4 k_{2} T^{-\beta}\right) e^{2 T^{\beta}}(M(T) u(T), u(T)) \leqq 0 .
$$

Thus letting $T \rightarrow \infty$ in (2.13), and increasing $\beta$, if necessary, we conclude that $u \equiv 0$ on $[2, \infty)$.

REMARKS. (i) Hypothesis (2.11) is quite restrictive, but the differential inequality (2.9) is so general that an assumption of a rate of decay slower than (2.11) yields counterexamples. It also should be observed that no hypothesis is required on the asymptotic behavior of $N$, the antisymmetric part of $A$.

(ii) The conditions of symmetry and antisymmetry on $M$ and $N$, respectively, are actually stronger than is needed and are mainly for computational convenience in the proofs of Lemmas 1 and 2. In both Theorems 1 and 2 we could drop the requirement that $M$ be symmetric, provided that in (II) we replace the term $(d / d t)(M(t) u(t)$, $u(t))$ by $(d / d t) \operatorname{Re}(M(t) u(t), u(t))$. For Theorem 2 the second part of (2.10) would also be replaced by the condition

$$
\varlimsup_{t \rightarrow \infty} e^{2 t^{\beta}} \operatorname{Re}(M(t) u(t), u(t)) \leqq 0 .
$$


The antisymmetry of $N$ was used in the proofs of Lemmas 1 and 2 only to eliminate the integral

$$
-2 \int \rho^{\prime} \operatorname{Re}(N v, v) d t
$$

by use of the antisymmetry condition

$$
\operatorname{Re}(N(t) w, w)=0,
$$

for all $t \in(0, T)$ and $w \in D_{t}$. (See equation (2.3).) We could weaken this antisymmetry requirement and still obtain acceptable bounds on (2.14) by replacing it with an estimate of the form

$$
\operatorname{Re}(N(t) w, w) \leqq \gamma_{B}\|w\|^{2}
$$

in the case of Theorem 1 , and by

$$
\operatorname{Re}(N(t) w, w) \geqq-\gamma_{6}(t)\|w\|^{2}
$$

in the case of Theorem 2. In Theorem 2 we would also require $\gamma_{\theta}(t)=0\left(t^{-1}\right)$ as $t \rightarrow+\infty$. Under these weaker requirements on $M$ and $N$ the proofs of Theorems 1 and 2 and their lemmas would follow as before with only minor and obvious changes.

3. Second order linear operators. As in $\S 2, H$ denotes a complex Hilbert space and $A=A(t)$ a linear operator on $H$ which, in general, is unbounded. We suppose the operator $A$ is decomposable into

$$
A(t)=M(t)+N(t)
$$

where $M$ is symmetric, $N$ is antisymmetric, and $D_{t}$, the domain of $M(t)$ and $N(t)$, is a linear manifold in $H$.

The function $u:[0, T) \rightarrow H$ is assumed to satisfy the following regularity conditions, the derivatives of $u$ being taken in the strong sense:

(iii) $u \in C^{1}([0, T) ; H) \cap C^{2}((0, T) ; H)$

(iv) $\left\|u^{\prime \prime}(t)\right\| \leqq K_{1}$ on $(0, T) ; K_{1}$ a constant

(v) $u(t) \in D_{t}, t \in(0, T)$.

In addition, the operators $M$ and $N$ in acting on $u$ are assumed to satisfy the regularity conditions:

(vi) $M u$ and $N u \in C((0, T) ; H)$

(vii) $\|M(t) u(t)\|,\|N(t) u(t)\| \leqq K_{2}$ on $(0, T), K_{2}$ a constant.

Let $\gamma_{i}, \sigma_{i}, \nu_{i}, i=1,2,3$, be nonnegative continuous functions on $(0, T)$. We define for all $t \in(0, T)$ the functions $F_{i}(t)=\gamma_{i}(t)\|u(t)\|^{2}+\sigma_{i}(t)\left\|u^{\prime}(t)\right\|^{2}+\nu_{i}(t)|(M(t) u(t), u(t))|, \quad i=1,2,3$. 
In addition to the continuous differentiability on $(0, T)$ of the functions $\operatorname{Re}\left(N(t) u(t), u^{\prime}(t)\right)$ and $(M(t) u(t), u(t))$, we assume the following three basic restrictions on the operators $M$ and $N$, which are required to hold for functions $u$ satisfying (iii)-(vii):
(A) $(d / d t) \operatorname{Re}\left(N(t) u(t), u^{\prime}(t)\right)-\operatorname{Re}\left(N(t) u(t), u^{\prime \prime}(t)\right) \geqq-F_{1}(t)$
(B) $\quad(d / d t)(M(t) u(t), u(t))-2 \operatorname{Re}\left(M(t) u(t), u^{\prime}(t)\right) \geqq-F_{2}(t)$
(C) $\operatorname{Re}(M(t) u(t), N(t) u(t)) \geqq-F_{3}(t)$.

Conditions (A) and (B) are useful for integration by parts, while condition (C) is a restriction on the relation between $M$ and $N$. For example, if $M$ and $N$ commute, then the left side of (C) vanishes and the condition is satisfied automatically.

For the second order differential operator

$$
L u=\frac{d^{2} u}{d t^{2}}-A u=u^{\prime \prime}-M u-N u
$$

the following weighted $L_{2}$-inequality is basic for the subsequent theorems.

LEMMA 3. Let $t_{0}, T_{0}$ be positive numbers with $t_{0}+T_{0}<1, T_{0}<$ T. Suppose $u$ satisfies (iii)-(vii) and that (A), (B), and (C) hold. Assume

$$
u(0)=u^{\prime}(0)=u\left(T_{0}\right)=u^{\prime}\left(T_{0}\right)=0 .
$$

Define $\tau=t+t_{0}$. Then for $\beta$ sufficiently large the following inequality holds:

$$
\begin{aligned}
\int_{0}^{T_{0}} e^{2 \tau-\beta} & {\left[\beta^{4} \tau^{-3 \beta-4}-2 \gamma_{1}-2 \beta \tau^{-\beta-1} \gamma_{2}-2 \gamma_{3}\right]\|u(t)\|^{2} d t } \\
& +\int_{0}^{T_{0}} e^{2 \tau}-\beta\left[\beta^{2} \tau^{-\beta-2}-2 \sigma_{1}-2 \beta \tau^{-\beta-1} \sigma_{2}-2 \sigma_{3}\right]\left\|u^{\prime}(t)\right\|^{2} d t \\
\leqq & \int_{0}^{T_{0}} e^{2 \tau}-\beta\|(L u)(t)\|^{2} d t \\
& +2 \int_{0}^{T_{0}} e^{2 \tau-\beta}\left[\nu_{1}+\beta \tau^{-\beta-1} \nu_{2}+\nu_{3}\right]|(M(t) u(t), u(t))| d t .
\end{aligned}
$$

Proof. We set $\varphi(t)=-\tau^{-\beta}=-\left(t+t_{0}\right)^{-\beta}$, and define $v=e^{-\varphi} u$. Then

$$
e^{-2 \varphi}\|L u\|^{2}=\left\|v^{\prime \prime}+2 \varphi^{\prime} v^{\prime}+\left(\phi^{\prime}\right)^{2} v+\phi^{\prime \prime} v-M v-N v\right\|^{2} .
$$

It follows that

$$
\begin{aligned}
\int_{0}^{T_{0}} e^{-2 \varphi} & \|L u\|^{2} d t \\
& \geqq 2 \operatorname{Re} \int_{0}^{T_{0}}\left(v^{\prime \prime}+\left(\varphi^{\prime}\right)^{2} v-M v, 2 \varphi^{\prime} v^{\prime}+\phi^{\prime \prime} v-N v\right) d t .
\end{aligned}
$$


With all integrations from 0 to $T_{0}$, we have

$$
\begin{aligned}
\int e^{-2 \varphi}\|L u\|^{2} d t \geqq 4 \operatorname{Re} \int \varphi^{\prime}\left(v^{\prime \prime}, v^{\prime}\right)+2 \operatorname{Re} \int \phi^{\prime \prime}\left(v^{\prime \prime}, v\right) \\
+4 \operatorname{Re} \int\left(\varphi^{\prime}\right)^{3}\left(v, v^{\prime}\right)+2 \int\left(\varphi^{\prime}\right)^{2} \varphi^{\prime \prime}\|v\|^{2}-2 \operatorname{Re} \int\left(v^{\prime \prime}, N v\right) \\
\quad-4 \operatorname{Re} \int \varphi^{\prime}\left(M v, v^{\prime}\right)-2 \int \varphi^{\prime \prime}(M v, v)+2 \operatorname{Re} \int(M v, N v) \\
\equiv I_{1}+I_{2}+\cdots+I_{8} .
\end{aligned}
$$

Using integration by parts in $I_{1}, I_{2}$, and $I_{3}$, we find

$$
\begin{aligned}
I_{1} & =2 \int \varphi^{\prime}\left(v^{\prime}, v^{\prime}\right)^{\prime}=-2 \int \rho^{\prime \prime}\left\|v^{\prime}\right\|^{2} \\
I_{2} & =2 \operatorname{Re} \int \varphi^{\prime \prime}\left[\left(v^{\prime}, v\right)^{\prime}-\left(v^{\prime}, v^{\prime}\right)\right]=-2 \operatorname{Re} \int \phi^{\prime \prime \prime}\left(v^{\prime}, v\right)-2 \int \phi^{\prime \prime}\left\|v^{\prime}\right\|^{2} \\
& =\int \varphi^{(i v)}\|v\|^{2}-2 \int \phi^{\prime \prime}\left\|v^{\prime}\right\|^{2} \\
I_{3} & =2 \int\left(\varphi^{\prime}\right)^{3}(v, v)^{\prime}=-6 \int\left(\varphi^{\prime}\right)^{2} \varphi^{\prime \prime}\|v\|^{2} .
\end{aligned}
$$

Thus we obtain

$$
\begin{aligned}
I_{1}+I_{2}+I_{3}+I_{4}= & -4 \int \phi^{\prime \prime}\left\|v^{\prime}\right\|^{2}+\int \varphi^{(i v)}\|v\|^{2} \\
& -4 \int\left(\phi^{\prime}\right)^{2} \varphi^{\prime \prime}\|v\|^{2} .
\end{aligned}
$$

Since $\varphi^{\prime \prime}<0$, we see that

$$
\begin{aligned}
-4 \varphi^{\prime \prime}\left\|v^{\prime}\right\|^{2} & =-4 \varphi^{\prime \prime} e^{-2 \varphi}\left\|u^{\prime}-\varphi^{\prime} u\right\|^{2} \\
& \geqq-4 \varphi^{\prime \prime} e^{-2 \varphi}\left[\frac{1}{4}\left\|u^{\prime}\right\|^{2}-\frac{1}{3}\left(\varphi^{\prime}\right)^{2}\|u\|^{2}\right] .
\end{aligned}
$$

Hence

$$
\begin{aligned}
I_{1}+I_{2}+I_{3}+I_{4} \geqq & -\int e^{-2 \varphi} \varphi^{\prime \prime}\left\|u^{\prime}\right\|^{2} \\
& +\int e^{-2 \varphi}\left[\varphi^{(i v)}-\frac{8}{3}\left(\phi^{\prime}\right)^{2} \varphi^{\prime \prime}\right]\|u\|^{2} .
\end{aligned}
$$

To estimate $I_{5}$ we employ (A) and get

$$
\begin{aligned}
I_{5} & =-2 \operatorname{Re} \int e^{-2 \varphi}\left(u^{\prime \prime}-2 \varphi^{\prime} u^{\prime}+\left(\varphi^{\prime}\right)^{2} u-\phi^{\prime \prime} u, N u\right) \\
& =-2 \operatorname{Re} \int e^{-2 \varphi}\left(u^{\prime \prime}, N u\right)+4 \operatorname{Re} \int e^{-2 \varphi} \varphi^{\prime}\left(u^{\prime}, N u\right)
\end{aligned}
$$




$$
\begin{aligned}
& \geqq-2 \int e^{-2 \varphi} \frac{d}{d t}\left[\operatorname{Re}\left(u^{\prime}, N u\right)\right]-2 \int e^{-2 \varphi} F_{1}(t)+4 \operatorname{Re} \int e^{-2 \varphi} \varphi^{\prime}\left(u^{\prime}, N u\right) \\
& =-2 \int e^{-2 \varphi} F_{1}(t) .
\end{aligned}
$$

We apply (B) to $I_{6}$ and take into account that $\varphi^{\prime}>0$ to obtain

$$
\begin{aligned}
I_{6} & =-4 \operatorname{Re} \int e^{-2 \varphi} \phi^{\prime}\left(M u, u^{\prime}-\varphi^{\prime} u\right) \\
& \geqq-2 \int e^{-2 \varphi} \phi^{\prime}\left[\frac{d}{d t}(M u, u)+F_{2}(t)\right]+4 \int e^{-2 \varphi}\left(\varphi^{\prime}\right)^{2}(M u, u) \\
& =2 \int e^{-2 \varphi} \varphi^{\prime \prime}(M u, u)-2 \int e^{-2 \varphi} \phi^{\prime} F_{2}(t) .
\end{aligned}
$$

Combining $I_{6}$ and $I_{7}$, we have

$$
I_{6}+I_{7} \geqq-2 \int e^{-2 \varphi} \varphi^{\prime} F_{2}(t) .
$$

Finally, we apply (C) to $I_{8}$ and find

$$
I_{8} \geqq-2 \int e^{-2 \varphi} F_{3}(t)
$$

Thus,

$$
\begin{aligned}
\int e^{-2 \varphi}\|L u\|^{2} \geqq \sum_{i=1}^{8} I_{i} \\
\geqq \int e^{-2 \varphi}\|u\|^{2}\left[-\frac{8}{3}\left(\varphi^{\prime}\right)^{2} \varphi^{\prime \prime}+\varphi^{(i v)}-2 \gamma_{1}-2 \varphi^{\prime} \gamma_{2}-2 \gamma_{3}\right] \\
\quad+\int e^{-2 \varphi}\left\|u^{\prime}\right\|^{2}\left[-\varphi^{\prime \prime}-2 \sigma_{1}-2 \varphi^{\prime} \sigma_{2}-2 \sigma_{3}\right] \\
\quad-2 \int e^{-2 \varphi}|(M u, u)|\left[\nu_{1}+\varphi^{\prime} \nu_{2}+\nu_{3}\right] .
\end{aligned}
$$

Substitution of $\varphi=-\tau^{-\beta}$ into (3.3) and the selection of $\beta$ sufficiently large yields (3.1).

Lemma 3 provides results on the uniqueness of the Cauchy problem for second order equations and inequalities in which $L u$ is the principal part. The next theorem does not impose bounds on the symmetric form $(M u, u)$.

THEOREM 3. Suppose $u$ satisfies (iii)-(vii) and that $M$ and $N$ satisfy conditions (A), (B), (C). Suppose also that $\gamma_{i}, \sigma_{i}$ are bounded and that $\nu_{i}=0, i=1,2,3$. Let $u$ satisfy the differential inequality

$$
\|(L u)(t)\|^{2} \leqq C_{0}\left[\omega(t)+\int_{0}^{t} \omega(s) d s\right], \quad t \in(0, T),
$$


where $C_{0}$ is a positive constant and

$$
\omega(t) \equiv\|u(t)\|^{2}+\left\|u^{\prime}(t)\right\|^{2} .
$$

If $u(0)=u^{\prime}(0)=0$, then $u \equiv 0$ on $[0, T)$.

Proof. For $\beta$ sufficiently large, inequality (3.1) takes the form

$$
\begin{gathered}
\frac{1}{2} \int_{0}^{T_{0}} e^{2 \tau-\beta}\left[\beta^{4} \tau^{-3 \beta-4}\|u\|^{2}+\beta^{2} \tau^{-\beta-2}\left\|u^{\prime}\right\|^{2}\right] d t \\
\leqq \int_{0}^{T_{0}} e^{2 \tau-\beta}\|L u\|^{2} d t
\end{gathered}
$$

provided $u$ also satisfies $u\left(T_{0}\right)=u^{\prime}\left(T_{0}\right)=0$. We introduce the function $\zeta(t)$ as in the proof of Theorem 1 and follow exactly the same procedure used there to conclude that $u \equiv 0$.

The next result allows us to weaken the hypotheses considerably by requiring only that the functions $\nu_{i}, i=1,2,3$, are bounded. However, we must then impose a one-sided bound on $(M u, u)$. Specifically, we employ one of the conditions

$\left(\mathrm{D}_{1}\right)$ On $(0, T), u$ satisfies

$$
(M(t) u(t), u(t)) \leqq \gamma_{4}(t)\|u(t)\|^{2}+\sigma_{4}(t)\left\|u^{\prime}(t)\right\|^{2} .
$$

$\left(D_{2}\right) \quad$ On $(0, T), u$ satisfies

$$
(M(t) u(t), u(t)) \geqq-\gamma_{4}(t)\|u(t)\|^{2}-\sigma_{4}(t)\left\|u^{\prime}(t)\right\|^{2},
$$

where $\gamma_{4}, \sigma_{4}$ are nonnegative continous functions on $(0, T)$.

Theorem 4. Suppose $u$ satisfies (iii)-(vii) and that $M$ and $N$ satisfy conditions (A), (B), (C), with $\gamma_{i}, \sigma_{i}, \nu_{i}, i=1,2,3$, bounded on $(0, T)$. Also suppose that either $\left(\mathrm{D}_{1}\right)$ or $\left(\mathrm{D}_{2}\right)$ holds with $\gamma_{4}, \sigma_{4}$ bounded functions. Assume that $u$ satisfies the differential inequality

$$
\|(L u)(t)\|^{2} \leqq C_{1}\left[\mu(t)+\int_{0}^{t} \mu(s) d s\right], t \in(0, T),
$$

where $C_{1}$ is a positive constant and

$$
\mu(t) \equiv\|u(t)\|^{2}+\left\|u^{\prime}(t)\right\|^{2}+|(M(t) u(t), u(t))| .
$$

If $u(0)=u^{\prime}(0)=0$, then $u \equiv 0$ on $[0, T)$.

Proof. We write

$$
\begin{aligned}
|(M u, u)| & =\left|\gamma_{4}\|u\|^{2}+\sigma_{4}\left\|u^{\prime}\right\|^{2}-(M u, u)-\gamma_{4}\|u\|^{2}-\sigma_{4}\left\|u^{\prime}\right\|^{2}\right| \\
& \leqq\left|\gamma_{4}\|u\|^{2}+\sigma_{4}\left\|u^{\prime}\right\|^{2}-(M u, u)\right|+\gamma_{4}\|u\|^{2}+\sigma_{4}\left\|u^{\prime}\right\|^{2} .
\end{aligned}
$$


If $\left(D_{1}\right)$ holds, then

$$
|(M u, u)| \leqq 2 \gamma_{4}\|u\|^{2}+2 \sigma_{4}\left\|u^{\prime}\right\|^{2}-(M u, u) .
$$

In a similar way, if $\left(D_{2}\right)$ holds, we have

$$
|(M u, u)| \leqq 2 \gamma_{4}\|u\|^{2}+2 \sigma_{4}\left\|u^{\prime}\right\|^{2}+(M u, u) .
$$

If either $\left(D_{1}\right)$ or $\left(D_{2}\right)$ holds and with $\varphi=-\tau^{-\beta}$, the above inequalities yield

$$
\begin{aligned}
& \int_{0}^{T_{0}} e^{-2 \varphi} \Phi^{\prime}|(M u, u)| d t \\
& \quad \leqq\left|\int_{0}^{T_{0}} e^{-2 \varphi} \varphi^{\prime}(M u, u) d t\right|+2 \int_{0}^{T_{0}} e^{-2 \varphi} \varphi^{\prime}\left[\gamma_{4}\|u\|^{2}+\sigma_{4}\left\|u^{\prime}\right\|^{2}\right] d t
\end{aligned}
$$

We have the identity

$$
\begin{aligned}
& \int e^{-2 \varphi} \varphi^{\prime}(M u, u)=\int e^{-2 \varphi} \varphi^{\prime} \operatorname{Re}\left(-L u+u^{\prime \prime}-N u, u\right) \\
& =-\int e^{-2 \varphi} \varphi^{\prime} \operatorname{Re}(L u, u)+\int e^{-2 \varphi} \varphi^{\prime} \operatorname{Re}\left(u^{\prime \prime}, u\right) \equiv J_{1}+J_{2} .
\end{aligned}
$$

For $J_{1}$ we use the elementary estimate

$$
\left|\varphi^{\prime}(L u, u)\right| \leqq\|L u\|^{2}+\frac{1}{4}\left(\varphi^{\prime}\right)^{2}\|u\|^{2}
$$

to get

$$
\left|J_{1}\right| \leqq \int e^{-2 \varphi}\left[\|L u\|^{2}+\frac{1}{4}\left(\phi^{\prime}\right)^{2}\|u\|^{2}\right]
$$

For $J_{2}$ we integrate by parts twice and obtain

$$
J_{2}=\int e^{-2 \varphi}\left[\frac{1}{2} \phi^{\prime \prime \prime}-3 \varphi^{\prime} \varphi^{\prime \prime}+2\left(\varphi^{\prime}\right)^{3}\right]\|u\|^{2}-\int e^{-2 \varphi} \varphi^{\prime}\left\|u^{\prime}\right\|^{2} .
$$

Thus we find

$$
\begin{aligned}
& \left|\int e^{-2 \varphi} \varphi^{\prime}(M u, u)\right| \leqq \int e^{-2 \varphi}\|L u\|^{2} \\
& \quad+\int e^{-2 \varphi}\left[\frac{1}{4}\left(\varphi^{\prime}\right)^{2}+\left|\frac{1}{2} \varphi^{\prime \prime \prime}-3 \varphi^{\prime} \varphi^{\prime \prime}+2\left(\varphi^{\prime}\right)^{3}\right|\right]\|u\|^{2} \\
& \quad+\int e^{-2 \varphi} \varphi^{\prime}\left\|u^{\prime}\right\|^{2} .
\end{aligned}
$$

We now substitute (3.7) into the right side of (3.6) and compute the derivatives of $\varphi$ to find for sufficiently large $\beta$, 


$$
\begin{aligned}
& \beta \int e^{2 \tau^{-\beta}} \tau^{-\beta-1}|(M u, u)| \leqq \int e^{2 \tau^{-\beta}}\|L u\|^{2} \\
& +\int e^{2 \tau-\beta}\left[6 \beta^{3} \tau^{-3 \beta-3}+2 \beta \tau^{-\beta-1} \gamma_{4}\right]\|u\|^{2} \\
& \quad+\int e^{2 \tau-\beta}\left[\beta \tau^{-\beta-1}+2 \beta \tau^{-\beta-1} \sigma_{4}\right]\left\|u^{\prime}\right\|^{2} .
\end{aligned}
$$

We multiply (3.8) by $\beta^{1 / 2}$ and add the result to (3.1) getting

$$
\begin{aligned}
\int_{0}^{T_{0}} e^{2 \tau}-\beta & {\left[\beta^{4} \tau^{-3 \beta-4}-6 \beta^{7 / 2} \tau^{-3 \beta-3}-2\left(\gamma_{1}+\gamma_{3}\right)-2 \beta \tau^{-\beta-1} \gamma_{2}\right.} \\
& \left.-2 \beta^{3 / 2} \tau^{-\beta-1} \gamma_{4}\right]\|u\|^{2}+\int_{0}^{T_{0}} e^{2 \tau-\beta}\left[\beta^{2} \tau^{-\beta-2}-2\left(\sigma_{1}+\sigma_{3}\right)\right. \\
& \left.-2 \beta \tau^{-\beta-1} \sigma_{2}-\beta^{3 / 2}\left(1+2 \sigma_{4}\right) \tau^{-\beta-1}\right]\left\|u^{\prime}\right\|^{2} \\
& +\int_{0}^{T_{0}} e^{2 \tau-\beta}\left[\beta^{3 / 2} \tau^{-\beta-1}-2\left(\nu_{1}+\nu_{3}\right)-2 \beta \nu_{2} \tau^{-\beta-1}\right]|(M u, u)| \\
\leqq & \int_{0}^{T_{0}} e^{2 \tau-\beta}\left(1+\beta^{1 / 2}\right)\|L u\|^{2} .
\end{aligned}
$$

Since the fixed functions $\gamma_{i}, \sigma_{i}, \nu_{i}$ are bounded, we may choose $\beta$ so large in (3.9) that the following simplified version is valid:

$$
\begin{aligned}
& \frac{1}{2} \beta^{4} \int_{0}^{T_{0}} e^{2 \tau}-\beta \tau^{-3 \beta-4}\|u\|^{2}+\frac{1}{2} \beta^{2} \int_{0}^{T_{0}} e^{2 \tau-\beta} \tau^{-\beta-2}\left\|u^{\prime}\right\|^{2} \\
& \quad+\frac{1}{2} \beta^{3 / 2} \int_{0}^{T_{0}} e^{2 \tau-\beta} \tau^{-\beta-1}|(M u, u)| \leqq 2 \beta^{1 / 2} \int_{0}^{T_{0}} e^{2 \tau-\beta}\|L u\|^{2} .
\end{aligned}
$$

To complete the proof, we introduce the function $\zeta(t)$ and follow the same procedure as in the proof of Theorem 1. We omit the details.

We now obtain a result for the asymptotic behavior of solutions of second order differential inequalities which is analogous to that obtained in Section 2 for first order inequalities. We require the following regularity conditions:

(viii) $u \in C^{2}((0, \infty) ; H)$

(ix) $u(t) \in D_{t}$ for all $t \in(0, \infty)$

( $\mathrm{x}) \quad M u$ and $N u \in C((0, \infty) ; H)$.

We further require conditions (A) and (C) with $T=+\infty$, but Condition (B) is replaced by the following conditions, which hold for functions $u$ satisfying (viii)-( $\mathbf{x})$ :

$\left(\mathrm{B}_{1}\right) \quad(M u, u)$ is continuously differentiable for $t \in(0, \infty)$

$\left(\mathrm{B}_{2}\right) \quad(d / d t)(M(t) u(t), u(t))-2 \operatorname{Re}\left(M(t) u(t), u^{\prime}(t)\right) \leqq \gamma_{2}(t)\|u(t)\|^{2}+$ $\sigma_{2}(t)\left\|u^{\prime}(t)\right\|^{2}+\nu_{2}(t)|(M(t) u(t), u(t))|$.

LEMmA 4. Suppose that $u$ satisfies (viii)-(x), that (A) and (C) hold with $T=+\infty$, and that $M$ satisfies $\left(\mathrm{B}_{1}\right)$ and $\left(\mathrm{B}_{2}\right)$. If $u(1)=$ 
$u^{\prime}(1)=0$, then for $\beta$ sufficiently large and for all $T>1$, the following inequality holds:

$$
\begin{aligned}
& \int_{1}^{T}\left[\beta^{4} t^{3 \beta-4}-2 \gamma_{1}(t)-2 \beta t^{\beta-1} \gamma_{2}(t)-2 \gamma_{3}(t)\right] e^{2 t \beta}\|u(t)\|^{2} d t \\
& \quad+\int_{1}^{T}\left[\frac{1}{2} \beta^{2} t^{\beta-2}-2 \sigma_{1}(t)-2 \beta t^{\beta-1} \sigma_{2}(t)-2 \sigma_{3}(t)\right] e^{2 t^{\beta}}\left\|u^{\prime}(t)\right\|^{2} d t \\
& \leqq e^{2 T \beta}\left[9 \beta^{3} T^{3 \beta}\|u(T)\|^{2}+2 \beta^{2} T^{\beta}\left\|u^{\prime}(T)\right\|^{2}+2 \operatorname{Re}\left(u^{\prime}(T),\right.\right. \\
&\left.\quad N(T) u(T))-2 \beta T^{\beta-1}(M(T) u(T), u(T))\right] \\
&+2 \int_{1}^{T}\left[\nu_{1}(t)+\beta t^{\beta-1} \nu_{2}(t)+\nu_{3}(t)\right] e^{2 t \beta}|(M(t) u(t), u(t))| d t \\
&+\int_{1}^{T} e^{2 t \beta}\|L u(t)\|^{2} d t .
\end{aligned}
$$

Proof. We proceed as in the proof of Lemma 3 except that we integrate from 1 to $T$ and choose $\varphi=-t^{\beta}$. The integrand is the same as in (3.2) but we observe that $\phi^{(k)}<0$ for all positive integers $k$. When evaluating the integrals $I_{1}, \cdots, I_{8}$ in (3.2), the evaluations at the upper limit must be taken into account since $u$ does not necessarily vanish for $t=T$. Thus we find

$$
\begin{aligned}
I_{1}= & 2 \varphi^{\prime}(T)\left\|v^{\prime}(T)\right\|^{2}-2 \int_{1}^{T} \phi^{\prime \prime}\left\|v^{\prime}\right\|^{2} \\
I_{2}= & 2 \operatorname{Re} \varphi^{\prime \prime}(T)\left(v^{\prime}(T), v(T)\right)-\varphi^{\prime \prime}(T)\|v(T)\|^{2} \\
& +\int_{1}^{T} \varphi^{(i v)}\|v\|^{2}-2 \int_{1}^{T} \phi^{\prime \prime}\left\|v^{\prime}\right\|^{2} \\
I_{3}= & 2\left(\varphi^{\prime}(T)\right)^{3}\|v(T)\|^{2}-6 \int_{1}^{T}\left(\varphi^{\prime}\right)^{2} \varphi^{\prime \prime}\|v\|^{2} .
\end{aligned}
$$

We use the same estimates on the integrals $I_{1}+\cdots+I_{4}$ as in Lemma 3. However, to simplify the non-integrated terms, we use the inequalities

$$
\begin{aligned}
2 \varphi^{\prime}\left\|v^{\prime}\right\|^{2} & =2 \varphi^{\prime} e^{-2 \varphi}\left\|u^{\prime}-\varphi^{\prime} u\right\|^{2} \\
& \geqq 4 \varphi^{\prime} e^{-2 \varphi}\left[\left\|u^{\prime}\right\|^{2}+\left(\varphi^{\prime}\right)^{2}\|u\|^{2}\right]
\end{aligned}
$$

and

$$
\begin{aligned}
2 \varphi^{\prime \prime} \operatorname{Re}\left(v^{\prime}, v\right) & =2 \phi^{\prime \prime} e^{-2 \varphi} \operatorname{Re}\left(u^{\prime}, u\right)-2 \varphi^{\prime} \varphi^{\prime \prime} e^{-2 \varphi}\|u\|^{2} \\
& \geqq e^{-2 \varphi}\left[\varphi^{\prime \prime}\left\|u^{\prime}\right\|^{2}+\varphi^{\prime \prime}\|u\|^{2}-2 \phi^{\prime} \varphi^{\prime \prime}\|u\|^{2}\right] .
\end{aligned}
$$

Hence

$$
\begin{aligned}
\sum_{i=1}^{4} I_{i} \geqq & -\int e^{-2 \varphi} \varphi^{\prime \prime}\left\|u^{\prime}\right\|^{2}+\int e^{-2 \varphi}\left[-\frac{8}{3}\left(\varphi^{\prime}\right)^{2} \varphi^{\prime \prime}+\varphi^{(i v)}\right]\|u\|^{2} \\
& +e^{-2 \varphi(T)}\|u(T)\|^{2}\left[6\left(\varphi^{\prime}(T)\right)^{3}-2 \varphi^{\prime}(T) \varphi^{\prime \prime}(T)\right] \\
& +e^{-2 \varphi(T)}\left\|u^{\prime}(T)\right\|^{2}\left[4 \varphi^{\prime}(T)+\varphi^{\prime \prime}(T)\right]
\end{aligned}
$$


The estimate for the integral $I_{5}$ in Lemma 3 becomes

$$
I_{5} \geqq-2 e^{-2 \varphi(T)} \operatorname{Re}\left(u^{\prime}(T), N(T) u(T)\right)-2 \int e^{-2 \varphi} F_{1}(t) .
$$

As for $I_{6}$, we apply $\left(B_{2}\right)$ and take into account that $\varphi^{\prime}<0$ to obtain (3.13) $I_{6}+I_{7} \geqq-2 \varphi^{\prime}(T) e^{-2 \varphi(T)}(M(T) u(T), u(T))+2 \int e^{-2 \varphi} \varphi^{\prime} F_{2}(t)$.

The term $I_{8}$ is unchanged so that

$$
I_{8} \geqq-2 \int e^{-2 \varphi} F_{3}(t) .
$$

The inequality (3.10) results from combining (3.11)-(3.14) for the integrals $I_{1}, \cdots, I_{8}$ and taking $\beta$ sufficiently large.

To establish restrictions on maximal rates of decay for solutions of second order equations and inequalities, we introduce the following conditions:

$$
\begin{array}{ll}
\left(\mathrm{E}_{1}\right) & \lim _{t \rightarrow \infty} e^{2 t \beta}\left[\|u(t)\|^{2}+\left\|u^{\prime}(t)\right\|^{2}\right]=0, \text { for all } \beta>0 \\
\left(\mathrm{E}_{2}\right) & \lim _{t \rightarrow \infty} e^{2 t^{\beta}} \operatorname{Re}\left(N(t) u(t), u^{\prime}(t)\right) \leqq 0, \text { for all } \beta>0 \\
\left(\mathrm{E}_{3}\right) & \lim _{t \rightarrow \infty} e^{2 t^{\beta}}(M(t) u(t), u(t)) \geqq 0, \text { for all } \beta>0 .
\end{array}
$$

We establish two results, the first of which requires no bound on $(M u, u)$.

THEOREM 5. Suppose that $u$ satisfies (viii)-(x), that (A) and (C) hold with $T=+\infty$, and that $M$ satisfies $\left(\mathrm{B}_{1}\right)$ and $\left(\mathrm{B}_{2}\right)$. Assume that $\nu_{i}=0, i=1,2,3$, and that $\sigma_{i}, \tau_{i}$ satisfy

$$
\begin{aligned}
& \sigma_{2}(t)=O\left(t^{-1}\right) \text { as } t \longrightarrow \infty \\
& \sigma_{1}(t), \sigma_{3}(t), \gamma_{1}(t), \gamma_{2}(t), \gamma_{3}(t)=O\left(t^{n}\right) \text { as } t \longrightarrow \infty \text { for some } n \geqq 0 .
\end{aligned}
$$

Let $u$ be a solution for all $t>0$ of the differential inequality

$$
\|(L u)(t)\|^{2} \leqq C_{2}(t)\left[\|u(t)\|^{2}+\left\|u^{\prime}(t)\right\|^{2}\right]
$$

where $C_{2}$ is a nonnegative continuous function with

$$
C_{2}(t)=O\left(t^{n}\right) \text { as } t \longrightarrow \infty \text {. }
$$

If $u$ satisfies $\left(\mathrm{E}_{1}\right),\left(\mathrm{E}_{2}\right)$, and $\left(\mathrm{E}_{3}\right)$, then $u \equiv 0$.

Proof. Under the additional assumption $u(1)=u^{\prime}(1)=0$, inequality (3.10) results. We introduce the function $\zeta(t)$ as in the proof of Theorem 2 and proceed along similar lines. The details are omitted. 
Theorem 6. Suppose that $u$ satisfies (viii)-(x), that (A) and (C) hold with $T=+\infty$, that $M$ satisfies $\left(\mathrm{B}_{1}\right)$ and $\left(\mathrm{B}_{2}\right)$, and that $\left(\mathrm{D}_{1}\right)$ or $\left(\mathrm{D}_{2}\right)$ holds with $T=+\infty$. Assume that

$$
\sigma_{2}, \nu_{2} \text { are } O\left(t^{-1}\right) \text { as } t \longrightarrow \infty,
$$

that $\sigma_{i}, \nu_{i}, \gamma_{j}$ are $O\left(t^{n}\right)$ as $t \longrightarrow \infty$ for some $n \geqq 0$ and $i=1,3, j=$ $1,2,3,4$, and that $\sigma_{4}$ is bounded. Let $u$ be a solution for all $t>0$ of the differential inequality

$$
\|(L u)(t)\|^{2} \leqq C_{3}(t)\left[\|u(t)\|^{2}+\left\|u^{\prime}(t)\right\|^{2}+|(M(t) u(t), u(t))|\right.
$$

where $C_{3}$ is a nonnegative continuous function with

$$
C_{3}(t)=O\left(t^{n}\right) \text { as } t \longrightarrow \infty \text {. }
$$

If $u$ satisfies $\left(\mathrm{E}_{1}\right),\left(\mathrm{E}_{2}\right)$, and $\left(\mathrm{E}_{3}\right)$, then $u \equiv 0$.

Proof. We first suppose $u(1)=u^{\prime}(1)=0$ and establish an extension of (3.10) under the hypothesis that either $\left(D_{1}\right)$ or $\left(D_{2}\right)$ holds. The technique is similar to that used in the proof of Theorem 4. We set $\varphi(t)=-t^{\beta}$ and define $\psi(t)=t^{\beta-2}$. Then if either $\left(D_{1}\right)$ or $\left(D_{2}\right)$ holds, we have (as in (3.6))

$$
\begin{aligned}
\int_{1}^{T} e^{-2 \varphi} \psi|(M u, u)| d t \leqq & \left|\int_{1}^{T} e^{-2 \varphi} \psi(M u, u) d t\right| \\
& +2 \int_{1}^{T} e^{-2 \varphi} \psi\left[\gamma_{4}\|u\|^{2}+\sigma_{4}\left\|u^{\prime}\right\|^{2}\right] d t .
\end{aligned}
$$

Also,

$$
\int e^{-2 \varphi} \psi(M u, u)=-\int e^{-2 \varphi} \psi \operatorname{Re}(L u, u)+\int e^{-2 \varphi} \psi \operatorname{Re}\left(u^{\prime \prime}, u\right) \equiv J_{3}+J_{4} .
$$

In $J_{3}$ we use the estimate

$$
|\psi(L u, u)| \leqq\|L u\|^{2}+\frac{1}{4} \psi^{2}\|u\|^{2} .
$$

In $J_{4}$ we integrate by parts and find

$$
\begin{aligned}
J_{4}= & -\int e^{-2 \varphi} \psi\left\|u^{\prime}\right\|^{2}+\int e^{-2 \varphi}\left[\frac{1}{2} \psi^{\prime \prime}-2 \varphi^{\prime} \psi^{\prime}-\varphi^{\prime \prime} \psi+2\left(\phi^{\prime}\right)^{2} \psi\right]\|u\|^{2} \\
& +e^{-2 \varphi(T)}\left[\psi(T)\left(u^{\prime}(T), u(T)\right)-\frac{1}{2} \psi^{\prime}(T)\|u(T)\|^{2}+\varphi^{\prime}(T) \psi(T)\|u(T)\|^{2}\right] .
\end{aligned}
$$

We estimate the quantity

$$
\left|\psi(T)\left(u^{\prime}(T), u(T)\right)\right| \leqq\left\|u^{\prime}(T)\right\|^{2}+\frac{1}{4}\left(\psi^{\prime}(T)\right)^{2}\|u(T)\|^{2}
$$


and insert this inequality into the right side of the above expression for $J_{4}$. Hence we obtain

$$
\begin{aligned}
& \int_{0}^{T} e^{2 t \beta} t^{\beta-2}|(M u, u)| d t \leqq \int_{1}^{T} e^{2 t \beta}\|L u\|^{2} \\
& +\int_{1}^{T} e^{2 t^{\beta}}\left[6 \beta^{2} t^{3 \beta-4}+2 t^{\beta-2} \gamma_{4}\right]\|u\|^{2} \\
& +\int_{1}^{T} e^{2 t \beta}\left[t^{\beta-2}+2 t^{\beta-2} \sigma_{4}\right]\left\|u^{\prime}\right\|^{2} \\
& +e^{2 T^{\beta}}\left[\left\|u^{\prime}(T)\right\|^{2}+2 \beta T^{2 \beta}\|u(T)\|^{2}\right] .
\end{aligned}
$$

We multiply (3.16) by $\beta^{3 / 2}$ and add the result to (3.10) getting

$$
\begin{aligned}
& \int_{1}^{T} e^{2 t \beta}\left[\beta^{4} t^{3 \beta-4}-2\left(\gamma_{1}+\gamma_{3}\right)-2 \beta t^{\beta-1} \gamma_{2}-6 \beta^{7 / 2} t^{3 \beta-4}-2 \beta^{3 / 2} t^{\beta-2} \gamma_{4}\right]\|u\|^{2} \\
& \quad+\int_{1}^{T} e^{2 t \beta}\left[\frac{1}{2} \beta^{2} t^{\beta-2}-2\left(\sigma_{1}+\sigma_{3}\right)-2 \beta t^{\beta-1} \sigma_{2}-\beta^{3 / 2} t^{\beta-2}-2 \beta^{3 / 2} t^{\beta-2} \sigma_{4}\right]\left\|u^{\prime}\right\|^{2} \\
& \quad+\int_{1}^{T} e^{2 t \beta}\left[\beta^{3 / 2} t^{\beta-2}-2\left(\nu_{1}+\nu_{3}\right)-2 \beta t^{\beta-2} \nu_{2}\right]|(M u, u)| \\
& \leqq \\
& \quad \int_{1}^{T} e^{2 t \beta}\left[1+\beta^{3 / 2}\right]\|L u\|^{2}+e^{2 T^{\beta}}\left[9 \beta^{3} T^{3 \beta}+2 \beta^{5 / 2} T^{2 \beta}\right]\|u(T)\|^{2} \\
& \quad+e^{2 T^{\beta}}\left[2 \beta^{2} T^{\beta}+\beta^{3 / 2}\right]\left\|u^{\prime}(T)\right\|^{2} \\
& \quad+e^{2 T^{\beta}}\left[2 \operatorname{Re}\left(N(T) u(T), u^{\prime}(T)\right)-2 \beta T^{\beta-1}(M(T) u(T), u(T))\right] .
\end{aligned}
$$

For $\beta$ sufficiently large (3.17) implies the inequality

$$
\begin{aligned}
\frac{1}{2} \int_{1}^{T} e^{2 t \beta} \beta^{4} t^{3 \beta-4}\|u\|^{2}+\frac{1}{4} \int_{1}^{T} e^{2 t \beta} \beta^{2} t^{\beta-2}\left\|u^{\prime}\right\|^{2} \\
\quad+\frac{1}{2} \int_{1}^{T} e^{2 t \beta} \beta^{3 / 2} t^{\beta-2}|(M u, u)| \leqq 2 \int_{1}^{T} e^{2 t^{\beta}} \beta^{3 / 2}\|L u\|^{2} \\
\quad+e^{2 T^{\beta}}\left[10 \beta^{3} T^{3 \beta}\|u(T)\|^{2}+3 \beta^{2} T^{\beta}\left\|u^{\prime}(T)\right\|^{2}\right. \\
\left.\quad+2 \operatorname{Re}\left(N(T) u(T), u^{\prime}(T)\right)-2 \beta T^{\beta-1}(M(T) u(T), u(T))\right] .
\end{aligned}
$$

The remainder of the proof follows as in the proof of Theorem 2, and we omit the details.

REMARKS. As in $\S 2$, we may relax the requirements of symmetry and anti-symmetry on $M$ and $N$, respectively, and the results of this section remain valid. We may drop the requirement that $M$ be symmetric if in all our conditions the symmetric form $(M u, u)$ is replaced by the expression $\operatorname{Re}(M u, u)$. This change would be made, for example, in conditions $(A)-(C),\left(D_{1}\right),\left(D_{2}\right),\left(E_{3}\right)$, and in the definition of $F_{i}(t)$. The condition of anti-symmetry on $N$ may be replaced by the weaker condition 


$$
\operatorname{Re}(N(t) u(t), u(t)) \leqq \gamma_{5}(t)\|u(t)\|^{2},
$$

where $\gamma_{5}$ is required to be bounded for Theorems 3 and 4, and $\gamma_{5}(t)=O\left(t^{n}\right)$ as $t \rightarrow+\infty$ for Theorems 5 and 6 . Under these weaker requirements on $M$ and $N$ the proofs of the results of this section require only minor and obvious changes.

4. First order nonlinear operators. Let $A=A(t)$ be an operator, in general nonlinear and unbounded, on a complex Hilbert space $H$. Let $D_{t}$ be the domain of $A(t)$ in $H$. We consider differential inequalities involving the first order operator

$$
(L u)(t)=u^{\prime}(t)-A(t) u(t) .
$$

We assume that solutions $u$ satisfy the following regularity conditions on $[0, T)$ (where $T$ may be $+\infty$ ):

$$
\begin{aligned}
& u \in C([0, T) ; H) \cap C^{1}((0, T) ; H) \\
& u(t) \in D_{t}, t \in(0, T) .
\end{aligned}
$$

For our first result the only requirement we impose on the operator $A$ is one of semi-boundedness. We assume either

$\left(\mathrm{P}_{1}\right) \quad \operatorname{Re}(A(t) u(t), u(t)) \leqq \gamma(t)\|u(t)\|^{2}, t \in(0, T)$ or

$\left(\mathrm{P}_{2}\right) \quad \operatorname{Re}(A(t) u(t), u(t)) \geqq-\gamma(t)\|u(t)\|^{2}, t \in(0, T)$,

where $\gamma$ is nonnegative and continuous on $(0, T)$. For certain first order inequalities we show that under hypothesis $\left(P_{1}\right)$ solutions of the Cauchy problem which vanish initially must vanish for all time, while under hypothesis $\left(\mathrm{P}_{2}\right)$ solutions cannot decay too rapidly, a result which implies that a solution which does not vanish initially can never vanish at any finite time.

THEOREM 7. Let $u$ satisfy conditions (4.1), and suppose that $u$ satisfies the differential inequality

$$
\|L u(t)\|^{2} \leqq K_{1}(t)\left[|\operatorname{Re}(A(t) u(t), u(t))|+K_{2}(t)\|u(t)\|^{2}\right], t \in(0, T) .
$$

Assume that $K_{1}, K_{2}$, and $\gamma$ are nonnegative and continuous on $(0, T)$ and are in $L_{1}(0, T)$; define

$$
\alpha(t)=\int_{0}^{t}\left[4 \gamma(s)+\frac{1}{2} K_{1}(s)+2 K_{2}(s)\right] d s, t \geqq 0 .
$$

(a) If condition $\left(\mathrm{P}_{1}\right)$ holds, then

$$
\|u(t)\|^{2} \leqq\|u(0)\|^{2} e^{\alpha(t)}, t \in[0, T) .
$$

(b) If condition $\left(\mathrm{P}_{2}\right)$ holds, then 


$$
\|u(t)\|^{2} \geqq\|u(0)\|^{2} e^{-\alpha(t)}, t \in[0, T) .
$$

Proof. We have, for $t \in(0, T)$,

$$
\begin{aligned}
& \frac{d}{d t}\|u(t)\|^{2}=2 \operatorname{Re}\left(u(t), u^{\prime}(t)\right) \\
& \quad=2 \operatorname{Re}(u(t), L u(t))+2 \operatorname{Re}(u(t), A(t) u(t)) \\
& \quad \leqq \delta(t)\|L u(t)\|^{2}+\delta(t)^{-1}\|u(t)\|^{2}+2 \operatorname{Re}(u(t), A(t) u(t))
\end{aligned}
$$

where $\delta(t)$ is any positive quantity. From (4.2) and $\left(\mathrm{P}_{1}\right)$ we find

$$
\begin{aligned}
\|L u\|^{2} & \leqq K_{1}\left|\gamma\|u\|^{2}-\operatorname{Re}(A u, u)-\gamma\|u\|^{2}\right|+K_{1} K_{2}\|u\|^{2} \\
& \leqq\left(2 \gamma K_{1}+K_{1} K_{2}\right)\|u\|^{2}-K_{1} \operatorname{Re}(A u, u) .
\end{aligned}
$$

At points where $K_{1}(t)>0$ we select $\delta(t)=2 K_{1}(t)^{-1}$ and obtain

$$
\frac{d}{d t}\|u(t)\|^{2} \leqq\left[4 \gamma(t)+\frac{1}{2} K_{1}(t)+2 K_{2}(t)\right]\|u(t)\|^{2}=\alpha^{\prime}(t)\|u(t)\|^{2} .
$$

At points where $K_{1}(t)=0$ we see from $(4.2)$ that $(L u)(t)=0$, and (4.4) follows from the first part of (4.3) and $\left(\mathrm{P}_{1}\right)$. Hence (4.4) holds for all $t \in(0, T)$, and integration yields

$$
\|u(t)\|^{2} \leqq\|u(0)\|^{2} e^{\alpha(t)} .
$$

Thus (a) is established. Part (b) is proved similarly, but with the inequalities reversed.

REMARK. Theorem 7 is an extension of known growth and decay results, the feature here being the inclusion of the term $|\operatorname{Re}(A(t) u(t), u(t))|$ in the right-hand side of the inequality (4.2).

If the nonlinear operator $A$ satisfies a differentiability condition, we may obtain similar results for more general first order inequalities. We perturb $A$ by another operator $B=B(t)$, also possibly nonlinear, with the domain $D_{t}$ of $B(t)$ the same as that of $A(t)$. The operator $L$ becomes

$$
(L u)(t)=u^{\prime}(t)-A(t) u(t)-B(t) u(t) .
$$

We require conditions (4.1) and either $\left(\mathrm{P}_{1}\right)$ or $\left(\mathrm{P}_{2}\right)$, and we assume

$$
\gamma(t) \leqq \gamma_{0}=\text { constant, } \gamma_{0} \geqq 0 \text { for all } t \in(0, T) .
$$

We assume the following differentiability condition on $A$ :

(Q) The function $\operatorname{Re}(A u, u)$ is continuously differentiable on $(0, T)$, and 


$$
\begin{aligned}
& \frac{d}{d t} \operatorname{Re}(A(t) u(t), u(t))-2 \operatorname{Re}\left(A(t) u(t), u^{\prime}(t)\right) \\
& \geqq-\gamma_{1}(t)\|u(t)\|^{2}-\nu_{1}(t)\|A(t) u(t)\|\|u(t)\| .
\end{aligned}
$$

We further assume for $t \in(0, T)$ the condition and either

(R) $\operatorname{Re}(A(t) u(t), B(t) u(t)) \geqq-\gamma_{2}(t)\|u(t)\|^{2}-\nu_{2}(t)\|A(t) u(t)\|\|u(t)\|$

$\left(\mathbf{S}_{1}\right) \quad \operatorname{Re}(B(t) u(t), u(t)) \leqq \gamma_{3}(t)\|u(t)\|^{2}+\nu_{3}(t)\|A(t) u(t)\|\|u(t)\|$

or

$\left(\mathbf{S}_{2}\right) \quad \operatorname{Re}\left(B(t) u(t), u_{i}^{(t)}\right) \geqq-\gamma_{3}(t)\|u(t)\|^{2}-\nu_{3}(t)\|A(t) u(t)\|\|u(t)\|$.

THEOREM 8. Suppose that $u$ and the operators $A$ and $B$ satisfy (4.1), (Q), and (R), and that (4.6) holds. Let Lu be given by (4.5), and suppose that $u$ satisfies the differential inequality

$$
\|L u(t)\|^{2} \leqq \gamma_{4}(t)\|u(t)\|^{2}+\nu_{4}(t)\|A(t) u(t)\|\|u(t)\|, t \in(0, T) .
$$

Assume that for $i=1, \cdots, 4$ each $\gamma_{i}$ and $\nu_{i}$ is continuous on $(0, T)$ and $\gamma_{i} \in L_{1}(0, T), \nu_{i} \in L_{2}(0, T)$. Define

$$
\beta(t) \equiv C_{0} \int_{0}^{t}\left[1+\sum_{i=1}^{4}\left(\gamma_{\imath}(s)+\nu_{i}(s)^{2}\right)\right] d s, t \in(0, T),
$$

for some suitably large positive constant $C_{0}$, and let $C \equiv \gamma_{0}+1$.

(a) Suppose $\left(\mathrm{P}_{1}\right)$ and $\left(\mathrm{S}_{1}\right)$ hold. Define

$$
\rho(t) \equiv c\|u(t)\|^{2}-\operatorname{Re}(A(t) u(t), u(t)), t \in(0, T) .
$$

Then

$$
\|u(t)\|^{2} \leqq \rho(t) \leqq e^{\beta(t)} \varliminf_{\varepsilon \rightarrow 0^{+}} \rho(\varepsilon), t \in(0, T)
$$

Hence if

$$
u(0)=0, \varlimsup_{t \rightarrow 0^{+}} \operatorname{Re}(A(t) u(t), u(t)) \geqq 0,
$$

then $u \equiv 0$ on $[0, T)$.

(b) Suppose $\left(\mathrm{P}_{2}\right)$ and $\left(\mathbf{S}_{2}\right)$ hold. In this case define

$$
\rho(t) \equiv c\|u(t)\|^{2}+\operatorname{Re}(A(t) u(t), u(t)) .
$$

Then

$$
\rho(t) \geqq e^{-\beta(t)} \varlimsup_{\varepsilon \rightarrow 0^{+}} \rho(\varepsilon) \geqq e^{-\beta(t)}\|u(0)\|^{2} .
$$

It follows that if either $u(0) \neq 0$ or $\varlimsup_{\lim _{t \rightarrow 0^{+}}} \operatorname{Re}(A(t) u(t), u(t))>0$, then $u(t) \neq 0$ for all $t$ in $(0, T)$.

Proof. We first prove (a). Let $c=\gamma_{0}+1$. We define a function $o$ on $(0, T)$ by 


$$
\rho(t) \equiv c\|u(t)\|^{2}-\operatorname{Re}(A(t) u(t), u(t)) .
$$

Then from $\left(\mathrm{P}_{1}\right)$ and $(4.6)$ we have

$$
\rho(t) \geqq\|u(t)\|^{2}, t \in(0, T) .
$$

Moreover, $\rho$ is continuously differentiable on $(0, T)$ and

$$
\rho^{\prime}=2 c \operatorname{Re}\left(u, u^{\prime}\right)-\frac{d}{d t} \operatorname{Re}(A u, u) .
$$

Using (4.5), $\left(\mathrm{P}_{1}\right),(4.7),\left(\mathrm{S}_{1}\right)$, and Cauchy's inequality, we obtain

$2 c \operatorname{Re}\left(u, u^{\prime}\right)=2 c \operatorname{Re}[(u, L u)+(u, A u)+(u, B u)]$

$$
\begin{aligned}
& \leqq\left(c^{2}\|u\|^{2}+\|L u\|^{2}+2 c\left(c\|u\|^{2}\right)+2 c \operatorname{Re}(u, B u)\right. \\
& \leqq 3 c^{2}\|u\|^{2}+\left(\gamma_{4}\|u\|^{2}+\nu_{4}\|A u\|\|u\|\right)+2 c\left(\gamma_{3}\|u\|^{2}+\nu_{3}\|A u\|\|u\|\right) \\
& \leqq\left(3 c^{2}+\gamma_{4}+2 c \gamma_{3}+\frac{3}{4} \nu_{4}^{2}+3 c^{2} \nu_{3}^{2}\right)\|u\|^{2}+\left(\frac{1}{3}+\frac{1}{3}\right)\|A u\|^{2} .
\end{aligned}
$$

Similarly, using (Q), (4.5), (R), (4.7), and Cauchy's inequality, we find

$$
\begin{aligned}
-\frac{d}{d t} & \operatorname{Re}(A u, u) \leqq-2 \operatorname{Re}\left(A u, u^{\prime}\right)+\gamma_{1}\|u\|^{2}+\nu_{1}\|A u\|\|u\| \\
\leqq & -2 \operatorname{Re}[(A u, L u)+(A u, A u)+(A u, B u)] \\
& +\left(\gamma_{1}+\frac{3}{4} \nu_{1}^{2}\right)\|u\|^{2}+\frac{1}{3}\|A u\|^{2} \\
\leqq & \left(\frac{1}{3}\|A u\|^{2}+3\|L u\|^{2}\right)-2\|A u\|^{2}+2\left(\gamma_{2}\|u\|^{2}+\nu_{2}\|A u\|\|u\|\right) \\
& +\left(\gamma_{1}+\frac{3}{4} \nu_{1}^{2}\right)\|u\|^{2}+\frac{1}{3}\|A u\|^{2} \\
\leqq & \left(\gamma_{1}+2 \gamma_{2}+3 \gamma_{4}+\frac{3}{4} \nu_{1}^{2}+3 \nu_{2}^{2}+\frac{27}{4} \nu_{4}^{2}\right)\|u\|^{2}-\frac{2}{3}\|A u\|^{2} .
\end{aligned}
$$

We substitute these estimates into (4.10) and find that there exists a constant $c_{0}>0$ such that

$$
\rho^{\prime} \leqq c_{0}\left[1+\sum_{i=1}^{4}\left(\gamma_{i}+\nu_{i}^{2}\right)\right]\|u\|^{2}, t \in(0, T) .
$$

We define the function

$$
\beta(t) \equiv c_{0} \int_{0}^{t}\left[1+\sum_{i=1}^{4}\left(\gamma_{i}(s)+\nu_{i}(s)^{2}\right)\right] d s, t \in(0, T) .
$$

Then

$$
\rho^{\prime}(t) \leqq \beta^{\prime}(t) \rho(t), \quad t \in(0, T),
$$


and integration yields, for any $\varepsilon>0$,

$$
\rho(t) \leqq e^{\beta(t)-\beta(\varepsilon)} \rho(\varepsilon), \quad 0<\varepsilon<t .
$$

Letting $\varepsilon$ tend to 0 , we obtain

$$
\|u(t)\|^{2} \leqq \rho(t) \leqq e^{\beta(t)} \varliminf_{\varepsilon \rightarrow 0^{+}} \rho(\varepsilon) .
$$

From (4.8) it follows that $\underline{\lim } \rho(\varepsilon)=0$ as $\varepsilon \rightarrow 0^{+}$, and hence $u \equiv 0$ on $[0, T)$.

In order to prove (b), instead of (4.9) we make the definition

$$
\rho(t) \equiv c\|u(t)\|^{2}+\operatorname{Re}(A(t) u(t), u(t)) .
$$

Making similar estimates as in the proof of (a), but with the inequalities reversed, we arrive at the inequality

$$
\rho^{\prime}(t) \geqq-\beta^{\prime}(t) \rho(t)
$$

which yields

$$
\rho(t) \geqq e^{-[\beta(t)-\beta(\varepsilon)]} \rho(\varepsilon), 0<\varepsilon<t .
$$

Letting $\varepsilon$ tend to 0 we find

$$
\rho(t) \geqq e^{-\beta(t)} \varlimsup_{\varepsilon \rightarrow 0^{+}} \rho(\varepsilon) \geqq e^{-\beta(t)}\|u(0)\|^{2} .
$$

Hence, if either $u(0) \neq 0$ or $\varlimsup_{t \rightarrow 0^{+}} \operatorname{Re}(A(t) u(t), u(t))>0$, then $\rho(t)>$ 0 for $t>0$, which implies $u(t) \neq 0$.

5. Second order nonlinear operators. In this section we apply the methods of $\S 4$ to nonlinear differential inequalities of the second order. We will show under rather general conditions that solutions with zero Cauchy data initially must vanish identically, and solutions with nonzero initial Cauchy data cannot have vanishing Cauchy data at a later time.

Again let $A(t)$ and $B(t)$ denote operators, both in general nonlinear and unbounded, on a complex Hilbert space $H$. Let $D_{t}$ be the domain in $H$ of $A(t)$ and $B(t)$, and let $L$ be the second order operator

$$
(L u)(t) \equiv u^{\prime \prime}(t)-A(t) u(t)-B(t) u(t) .
$$

We assume the regularity conditions

$$
\begin{aligned}
& u \in C^{1}([0, T) ; H) \cap C^{2}((0, T) ; H) \\
& u(t) \in D_{t}, t \in(0, T) .
\end{aligned}
$$

We impose on the operator $A$ the condition 
(T) $\operatorname{Re}(A(t) u(t), u(t)) \leqq \gamma\|u(t)\|^{2}+\sigma\left\|u^{\prime}(t)\right\|^{2}, t \in(0, T)$,

where $\gamma, \sigma$ are positive constants, with

$$
0<\sigma<1 \text {. }
$$

Let $\gamma_{i}, \sigma_{i}, \nu_{i}, i=1,2,3$, be positive and continuous functions on $(0, T)$. We require further that the function $\operatorname{Re}(A u, u)$ be continuously differentiable on $(0, T)$, and that one of the two following inequalities holds:

$$
\begin{aligned}
& \frac{d}{d t} \operatorname{Re}(A(t) u(t), u(t))-2 \operatorname{Re}\left(A(t) u(t), u^{\prime}(t)\right) \\
& \quad \geqq-\gamma_{1}(t)\|u(t)\|^{2}-\sigma_{1}(t)\left\|u^{\prime}(t)\right\|^{2}-\nu_{1}(t)|\operatorname{Re}(A(t) u(t), u(t))|
\end{aligned}
$$

or

$$
\begin{aligned}
& \frac{d}{d t} \operatorname{Re}(A(t) u(t), u(t))-2 \operatorname{Re}\left(A(t) u(t), u^{\prime}(t)\right) \\
& \quad \leqq \gamma_{1}(t)\|u(t)\|^{2}+\sigma_{1}(t)\left\|u^{\prime}(t)\right\|^{2}+\nu_{1}(t)|\operatorname{Re}(A(t) u(t), u(t))| .
\end{aligned}
$$

We also impose one of the conditions

$$
\begin{aligned}
\operatorname{Re}\left(u^{\prime}(t), B(t) u(t)\right) \leqq \gamma_{2}(t) & \|u(t)\|^{2}+\sigma_{2}(t)\left\|u^{\prime}(t)\right\|^{2} \\
& +\nu_{2}(t)|\operatorname{Re}(A(t) u(t), u(t))|
\end{aligned}
$$

or

$$
\begin{aligned}
\operatorname{Re}\left(u^{\prime}(t), B(t) u(t)\right) \geqq-\gamma_{2}(t) & \|u(t)\|^{2}-\sigma_{2}(t)\left\|u^{\prime}(t)\right\|^{2} \\
& -\nu_{2}(t)|\operatorname{Re}(A(t) u(t), u(t))| .
\end{aligned}
$$

We note that $(\mathrm{T})$ is a one-sided bound on $\operatorname{Re}(A u, u)$ which is satisfied if the more restrictive inequality

$$
\operatorname{Re}(A u, u) \leqq k\left(\|u\|^{2}+\|u\|\left\|u^{\prime}\right\|\right)
$$

holds for some positive constant $k$. The conditions $\left(\mathrm{U}_{1}\right)$ and $\left(\mathrm{U}_{2}\right)$, as in all such hypotheses, allow integration by parts. In fact, if $A$ is symmetric and has a derivative $A^{\prime}$, then these conditions are one-sided restrictions on the graph of $A^{\prime}$.

THEOREM 9. Suppose that $u$ satisfies conditions (5.2) and the differential inequality

$$
\begin{gathered}
\|L u(t)\|^{2} \leqq \gamma_{3}(t)\|u(t)\|^{2}+\sigma_{3}(t)\left\|u^{\prime}(t)\right\|^{2}+\nu_{3}(t)|\operatorname{Re}(A(t) u(t), u(t))|, \\
t \in(0, T) .
\end{gathered}
$$

Let $\gamma_{i}, \sigma_{i}, \nu_{i}, i=1,2,3$, be continuous positive functions in $L_{1}(0, T)$. Define 


$$
\begin{aligned}
& \rho(t) \equiv c\|u(t)\|^{2}+\left\|u^{\prime}(t)\right\|^{2}-\operatorname{Re}(A(t) u(t), u(t)), t \in(0, T), \\
& \beta(t) \equiv c_{0} \int_{0}^{t}\left[1+\sum_{i=1}^{3}\left(\gamma_{i}(s)+\sigma_{i}(s)+\nu_{i}(s)\right)\right] d s, t \in(0, T),
\end{aligned}
$$

where $c \equiv \gamma+1$, and $c_{0}$ is a suitably large positive constant.

(a) If $(\mathrm{T}),\left(\mathrm{U}_{1}\right)$, and $\left(\mathrm{V}_{1}\right)$ hold, then

$$
\|u(t)\|^{2}+(1-\sigma)\left\|u^{\prime}(t)\right\|^{2} \leqq \rho(t) \leqq e^{\beta(t)} \varliminf_{\varepsilon+0^{+}} \rho(\varepsilon), t \in(0, T) .
$$

Hence if

$$
u(0)=u^{\prime}(0)=0, \varlimsup_{t \rightarrow 0^{+}} \operatorname{Re}(A(t) u(t), u(t)) \geqq 0,
$$

then $u \equiv 0$ on $[0, T)$.

(b) If $(\mathrm{T}),\left(\mathrm{U}_{2}\right)$, and $\left(\mathrm{V}_{2}\right)$ hold, then

$$
\rho(t) \geqq e^{-\beta(t)} \varlimsup_{\varepsilon \rightarrow 0^{+}} \rho(\varepsilon) \geqq e^{-\beta(t)}\left[\|u(0)\|^{2}+(1-\sigma)\left\|u^{\prime}(0)\right\|^{2}\right], t \in(0, T) .
$$

It follows that if either $u(0) \neq 0, u^{\prime}(0) \neq 0$, or $\underline{\lim }_{t \rightarrow 0^{+}} \operatorname{Re}(A(t) u(t)$, $u(t))<0$, then $u(t)$ and $u^{\prime}(t)$ cannot vanish simultaneously for any $t$ in $(0, T)$.

Proof. We first prove (a). We let $c=\gamma+1$, and define a function $\rho$ on $(0, T)$ by

$$
\rho(t) \equiv c\|u(t)\|^{2}+\left\|u^{\prime}(t)\right\|^{2}-\operatorname{Re}(A(t) u(t), u(t)) .
$$

From (T) and (5.3) we obtain

$$
\rho(t) \geqq\|u(t)\|^{2}+(1-\sigma)\left\|u^{\prime}(t)\right\|^{2} .
$$

Moreover, $\rho$ is continuously differentiable on $(0, T)$, and

$$
\rho^{\prime}=2 c \operatorname{Re}\left(u, u^{\prime}\right)+2 \operatorname{Re}\left(u^{\prime}, u^{\prime \prime}\right)-\frac{d}{d t} \operatorname{Re}(A u, u) .
$$

Cauchy's inequality gives

$$
2 c \operatorname{Re}\left(u, u^{\prime}\right) \leqq c\|u\|^{2}+c\left\|u^{\prime}\right\|^{2} .
$$

Using $\left(\mathrm{V}_{1}\right),(5.4)$, and Cauchy's inequality we obtain

$$
\begin{aligned}
& 2 \operatorname{Re}\left(u^{\prime}, u^{\prime \prime}\right)=2 \operatorname{Re}\left[\left(u^{\prime}, L u\right)+\left(u^{\prime}, A u\right)+\left(u^{\prime}, B u\right)\right] \\
& \leqq\left(\left\|u^{\prime}\right\|^{2}+\|L u\|^{2}\right)+2 \operatorname{Re}\left(u^{\prime}, A u\right) \\
&+\left(2 \gamma_{2}\|u\|^{2}+2 \sigma_{2}\left\|u^{\prime}\right\|^{2}+2 \nu_{2}|\operatorname{Re}(A u, u)|\right) \\
& \leqq\left(2 \gamma_{2}+\gamma_{3}\right)\|u\|^{2}+\left(2 \sigma_{2}+\sigma_{3}+1\right)\left\|u^{\prime}\right\|^{2} \\
&+\left(2 \nu_{2}+\nu_{3}\right)|\operatorname{Re}(A u, u)|+2 \operatorname{Re}\left(u^{\prime}, A u\right) .
\end{aligned}
$$


Combining (5.9) $-(5.11)$ and $\left(\mathrm{U}_{1}\right)$ gives

$$
\begin{aligned}
\rho^{\prime} \leqq\left(c+\gamma_{1}+2 \gamma_{2}+\gamma_{3}\right)\|u\|^{2}+ & \left(1+c+\sigma_{1}+2 \sigma_{2}+\sigma_{3}\right)\left\|u^{\prime}\right\|^{2} \\
& +\left(\nu_{1}+2 \nu_{2}+\nu_{3}\right)|\operatorname{Re}(A u, u)| .
\end{aligned}
$$

We also have the estimate

$$
\begin{aligned}
|\operatorname{Re}(A u, u)| & =\left|c\|u\|^{2}+\left\|u^{\prime}\right\|^{2}-\operatorname{Re}(A u, u)-c\|u\|^{2}-\left\|u^{\prime}\right\|^{2}\right| \\
& \leqq \rho+c\|u\|^{2}+\left\|u^{\prime}\right\|^{2}
\end{aligned}
$$

and also from (5.8),

$$
\|u\|^{2} \leqq \rho,\left\|u^{\prime}\right\|^{2} \leqq(1-\sigma)^{-1} \rho .
$$

Hence, upon combining (5.12)-(5.14) we find that there exists a constant $c_{0}>0$ such that

$$
\rho^{\prime} \leqq c_{0}\left[1+\sum_{i=1}^{3}\left(\gamma_{i}+\sigma_{i}+\nu_{i}\right)\right] \rho \text {. }
$$

We define

$$
\beta(t) \equiv c_{0} \int_{0}^{t}\left[1+\sum_{i=1}^{3}\left(\gamma_{i}(s)+\sigma_{i}(s)+\nu_{i}(s)\right)\right] d s
$$

Then

$$
\rho^{\prime}(t) \leqq \beta^{\prime}(t) \rho(t), t \in(0, T),
$$

and integration yields, for $0<\varepsilon<t$,

$$
\rho(t) \leqq e^{\beta(t)-\beta(\varepsilon)} \rho(\varepsilon) \text {. }
$$

Letting $\varepsilon$ tend to 0 , we obtain

$$
\|u(t)\|^{2}+(1-\sigma)\left\|u^{\prime}(t)\right\|^{2} \leqq \rho(t) \leqq e^{\beta(t)} \varliminf_{\varepsilon \rightarrow 0^{+}} \rho(\varepsilon) .
$$

From (5.5) it follows that $\underline{\lim } \rho(\varepsilon)=0$ as $\varepsilon \rightarrow 0^{+}$, and hence $u \equiv 0$ on $[0, T)$.

The proof of (b) is similar to that of (a), but the inequalities are reversed. Instead of (5.13) we arrive at the inequality

$$
\rho^{\prime}(t) \geqq-\beta^{\prime}(t) \rho(t), \quad t \in(0, T),
$$

which yields

$$
\rho(t) \geqq e^{-[\beta(t)-\beta(\varepsilon)]} \rho(\varepsilon), \quad 0<\varepsilon<t
$$

and hence

$$
\rho(t) \geqq e^{-\beta(t)} \varlimsup_{\varepsilon \rightarrow 0^{+}} \rho(\varepsilon)
$$


If either $u(0) \neq 0, u^{\prime}(0) \neq 0$, or $\underline{\lim }_{t \rightarrow 0^{+}} \operatorname{Re}(A(t) u(t), u(t))<0$, then $\overline{\lim }_{\varepsilon \rightarrow 0^{+}} \rho(\varepsilon)>0$, and $\rho(t)>0$ for $t>0$. But $\rho(t)>0$ implies $u(t)$ and $u^{\prime}(t)$ cannot vanish simultaneously.

\section{REFERENCES}

1. S. Agmon and L. Nirenberg, Lower bounds and uniqueness theorems for solutions of differential equations in a Hilbert space, Comm. Pure Appl. Math., 20 (1967), 207-229.

2. R. J. Knops, H. A. Levine, and L. E. Payne, Non-existence, instability, and growth theorems for solutions of a class of abstract nonlinear equations with applications to nonlinear elastodynamics, Arch. Rational Mech. Anal., 55 (1974), 52-72.

3. R. J. Knops and L. E. Payne, Continuous data dependence for the equations of classical elastodynamics, Proc. Camb. Phil. Soc., 66 (1969), 481-491.

4. - Growth estimates for solutions of evolutionary equations in Hilbert space with applications in elastodynamics, Arch. Rational Mech. Anal., 41 (1971), 363-398.

5. H. A. Levine, Logarithmic convexity and the Cauchy problem for some abstract second order differential inequalities, J. Differential Equations, 8 (1970), 34-55.

6. —_ Logarithmic convexity, first order differential inequalities and some applications, Trans. Amer. Math. Soc., 152 (1970), 299-320.

7. - Some uniqueness and growth theorems in the Cauchy problem for $P u_{t t}+$ $M u_{t}+N u=0$ in Hilbert space, Math. Z., 126 (1972), 345-360.

8. - On the uniqueness of bounded solutions to $u^{\prime}(t)=A(t) u(t)$ and $u^{\prime \prime}(t)=$ $A(t) u(t)$ in Hilbert space, SIAM J. Math. Anal., 4, Nr. 2, (May 1973), 250-259.

9. - Some new uniqueness and continuous dependence results for evolutionary equations of indefinite type: the weighted energy method, J. Differential Equations, 19 (1975), 330-343.

10. A. C. Murray, Asymptotic behavior of solutions of hyperbolic inequalities, Trans. Amer. Math. Soc., 157 (1971), 279-296.

11. - Asymptotic behavior and uniqueness for an ultrahyperbolic equation with variable coefficients, J. Differential Equations, 23 (1977), 200-215.

12. A. C. Murray and M. H. Protter, The asymptotic behavior of second order systems of partial differential equations, J. Differential Equations, 13 (1973), 57-80.

13. H. Ogawa, Lower bounds for solutions of hyperbolic inequalities, Proc. Amer. Math. Soc., 16 (1965), 853-857.

14. Lower bounds for solutions of hyperbolic inequalities in expanding domains, J. Differential Equations, 13 (1973), 384-390.

15. M. H. Protter, Asymptotic behavior and uniqueness theorems for hyperbolic equations and inequalities, Proc. U.S.A.-U.S.S.R. Symposium on Partial Differential Equations, Novosibirsk (1963), 348-353.

Received April 15, 1981. The first author was supported in part by National Science Foundation grant MCS 78-01993, and by a fellowship from the Humboldt Foundation of West Germany while visiting at the Free University of Berlin. The second author was supported in part by NSF Grant MCS 79-05791.

\section{UNIVERSITY OF HAWAII}

AND

UNIVERSITY OF CALIFORNIA

Berkeley, CA 94720 


\section{PACIFIC JOURNAL OF MATHEMATICS}

\section{EDITORS}

DONALD BABBITT (Managing Editor)

University of California

Los Angeles, California 90024

\section{Hugo Rossi}

University of Utah

Salt Lake City, UT 84112

C. C. MOore and Arthur AGuS

University of California

Berkeley, CA 94720
J. DugundJI

Department of Mathematics University of Southern California Los Angeles, California 90007

R. FinN and J. Milgram Stanford University Stanford, California 94305

ASSOCIATE EDITORS
R. ARENS
E. F. BECKENBACH
B. H. NeumanN
F. WOLF
K. YOSHIDA

\section{SUPPORTING INSTITUTIONS}

UNIVERSITY OF ARIZONA

UNIVERSITY OF BRITISH COLUMBIA

CALIFORNIA INSTITUTE OF TECHNOLOGY

UNIVERSITY OF CALIFORNIA

MONTANA STATE UNIVERSITY

UNIVERSITY OF NEVADA, RENO

NEW MEXICO STATE UNIVERSITY

OREGON STATE UNIVERSITY
UNIVERSITY OF OREGON

UNIVERSITY OF SOUTHERN CALIFORNIA

STANFORD UNIVERSITY

UNIVERSITY OF HAWAII

UNIVERSITY OF TOKYO

UNIVERSITY OF UTAH

WASHINGTON STATE UNIVERSITY

UNIVERSITY OF WASHINGTON 


\section{Pacific Journal of Mathematics}

Vol. 99, No. $1 \quad$ May, 1982

Mariano Giaquinta, Jindrich Necas, O. John and J. Stará, On the

regularity up to the boundary for second order nonlinear elliptic systems . . 1

Siegfried Graf, Realizing automorphisms of quotients of product $\sigma$-fields . . 19

Alfred Washington Hales and Ernst Gabor Straus, Projective colorings . . . 31

Sandra Hayes, The weak Nullstellensatz for finite-dimensional complex

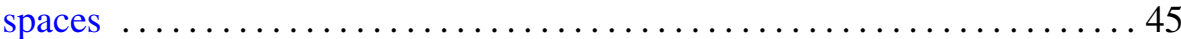

Gerald Norman Hile and Murray Harold Protter, The Cauchy problem

and asymptotic decay for solutions of differential inequalities in Hilbert

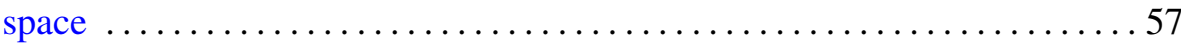

Robert D. Little, Projective space as a branched covering with orientable

branch set ......................................... 89

Jaroslav Mach, On the proximinality of Stone-Weierstrass subspaces . . . . . 997

John C. Morgan, II, On product bases ...................... 105

K. Balakrishna Reddy and P. V. Subrahmanyam, Altman's contractors

and fixed points of multivalued mappings . .................. 127

James Ted Rogers Jr., Decompositions of homogeneous continua . . . . . . . 137

Ahmed Ramzy Sourour, Characterization and order properties of

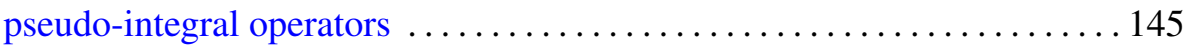

Robert Moffatt Stephenson Jr., Pseudocompact and Stone-Weierstrass

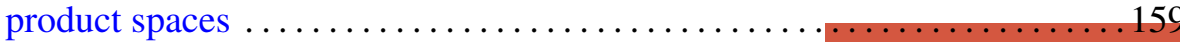

Bruce Stewart Trace, On attaching 3-handles to a 1-connected

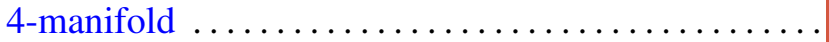

Akihito Uchiyama, The construction of certain BMO functions and the corona problem

Thomas Alva Whitehurst, An application of orthogonal polynomials to random walks ..............................

David J. Winter, Root locologies and idempotents of Lie and nonassociative algebras

William Robin Zame, The classification of uniform algebras on plane domains 